$41(2) \mid 2012$

Varia

\title{
Catégorisation des plantes et des entités étiologiques chez les Yanesha (piémont amazonien du Pérou)
}

Categorización vernacular de las plantas y entidades etiológicas de los yánesha de la selva central (Perú)

Vernacular categorization of the Yanesha plants and etiological agents in the high Peruvian Amazon

\section{Céline Valadeau}

\section{OpenEdition}

Journals

Édition électronique

URL : http://journals.openedition.org/bifea/1026

DOI : 10.4000/bifea.1026

ISSN : 2076-5827

Éditeur

Institut Français d'Études Andines

Édition imprimée

Date de publication : 1 juillet 2012

Pagination : 241-281

ISSN : 0303-7495

Référence électronique

Céline Valadeau, «Catégorisation des plantes et des entités étiologiques chez les Yanesha (piémont amazonien du Pérou) », Bulletin de l'Institut français d'études andines [En ligne], 41 (2) | 2012, mis en ligne le 01 décembre 2012, consulté le 27 novembre 2020. URL : http://journals.openedition.org/bifea/ 1026 ; DOI : https://doi.org/10.4000/bifea.1026

\section{(c)}

Les contenus du Bulletin de l'Institut français d'études andines sont mis à disposition selon les termes de la licence Creative Commons Attribution - Pas d'Utilisation Commerciale - Pas de Modification 4.0 International. 


\title{
Catégorisation des plantes et des entités étiologiques chez les Yanesha (piémont amazonien du Pérou)
}

\author{
Céline Valadeau*
}

\section{Résumé}

La compréhension des systèmes de catégorisation vernaculaires a intéressé bien des auteurs. Dans cette perspective, ce texte cherche à analyser à partir de données issues d'une étude ethnobotanique et de relevés ethnographiques l'univers végétal des Yanesha de Haute Amazonie péruvienne. Cette analyse porte principalement sur les végétaux utilisés lors des soins dispensés à la suite des affections dues à des entités étiologiques. Dans un premier temps, ce texte présente les entités étiologiques connues pour provoquer des états dégradés de bonne santé. Dans un deuxième temps, les plantes préconisées lors de telles affections sont décrites à travers leurs racines mythologiques puis grâce à une étude linguistique de leurs dénominations. La dernière partie du texte explique comment les catégorisations vernaculaires des entités étiologiques et des plantes se superposent dans un système logique. De nombreux liens de cohérence permettent alors de comprendre le fonctionnement du système nosologique Yanesha et les logiques de dénomination des plantes lors des évènements morbides.

Mots clés : plante, catégorisation vernaculaire, nosographie, mythes, Amazonie, Pérou, Yanesha

\section{Categorización vernacular de las plantas y entidades etiológicas de los yánesha de la selva central (Perú)}

\section{Resumen}

Los sistemas de categorización vernaculares interesaron a numerosos autores. Este artículo analiza los datos etnobotánicos y etnográficos del universo vegetal de los yánesha de la Selva Central peruana.

* Centre EREA - UMR 7186. CNRS - Bât. D, Haudricourt 7 rue Guy Môquet 94801 Villejuif cedex. Ex becaria del IFEA entre 2007 y 2009. Investigadora asociada desde 2010 y hasta 2012. E-mail : valadeau.celine@yahoo.fr 
Estudia las plantas conocidas por sanar las afecciones inducidas por entidades etiológicas, a las cuales se atribuye el deterioro de una buena salud. Por otra parte, las plantas son referidas a través de sus raíces mitológicas y de un estudio lingüístico de sus denominaciones. La última parte del texto explica cómo la clasificación vernacular de las entidades etiológicas y la de las plantas puede ser pensada en un mismo sistema lógico. Estas organizaciones clasificatorias se superponen y permiten tener una clave de entendimiento de la articulación del sistema nosológico yánesha y de las lógicas de denominación de las plantas utilizadas en estos acontecimientos mórbidos.

Palabras claves: planta, categorización vernacular, nosografía, mitos, Amazonía, Perú, yánesha

\title{
Vernacular categorization of the Yanesha plants and etiological agents in the high Peruvian Amazon
}

\begin{abstract}
Vernacular categorization systems have been studied for several years. In this article, the purpose is to analyze ethnobotanical and ethnographic data of the plants by the Yanesh of the high Peruvian Amazon in central Peru. The first focus of this study is to define the different known etiological agents. This enables us to identify the causes of a negative impact on health. In this context, healing plants are studied using myths and a linguistic study of plant names. The second focus of this article shows how the vernacular classification of etiologic entities and plants can be seen as constituting a single logical system. These systems of classification overlap and provide a key to understanding the articulation of the system by which the Yanesha classify illness sha and the logic underlying the naming of plants utilized in the event of illness.
\end{abstract}

Key words: plant, vernacular categorization, nosography, myths, Amazon, Peru, Yanesha

\section{INTRODUCTION}

Les Amuesha', groupe de filiation linguistique Arawak, résident en Haute Amazonie péruvienne au pieds des massifs San Carlos et Yanachaga-Chemillén (Région : Oxapampa, département : Pasco) et au bord des fleuves Huancabamba, Cacazú et Palcazú. Vivant essentiellement de la pêche, de la chasse et des activités de cueillette, leur territoire s'est délocalisé en direction des basses terres à cause des colonisations successives (Smith, 2004). Cette délocalisation ne fut pas très importante, leur territoire actuel reste proche des lieux relatés dans les mythes, mais elle définira toutefois deux zones d'occupation : une dite « de haute altitude» et l'autre « de basse altitude » (Santos-Granero et al., 2004). La première se définit par des forêts de haute altitude subtropicales humides et des forêts très humides nuageuses et la seconde par des forêts tropicales amazoniennes et subtropicales de transition.

Les Amuesha' se définirent comme Yanesha' au moment de l'avènement de la fédération Feconaya (Federación de Comunidades Nativas Yanesha) en 1980 (fig. 1). Cette redéfinition identitaire permit de donner une réelle signification au terme les représentant. Le pronom nominal ya- exprime la première personne du pluriel et -nesha' est un suffixe désignant un groupe d'êtres. Accolées, ces deux entités linguistiques signifient littéralement «nous, l'ensemble des êtres humains ». 


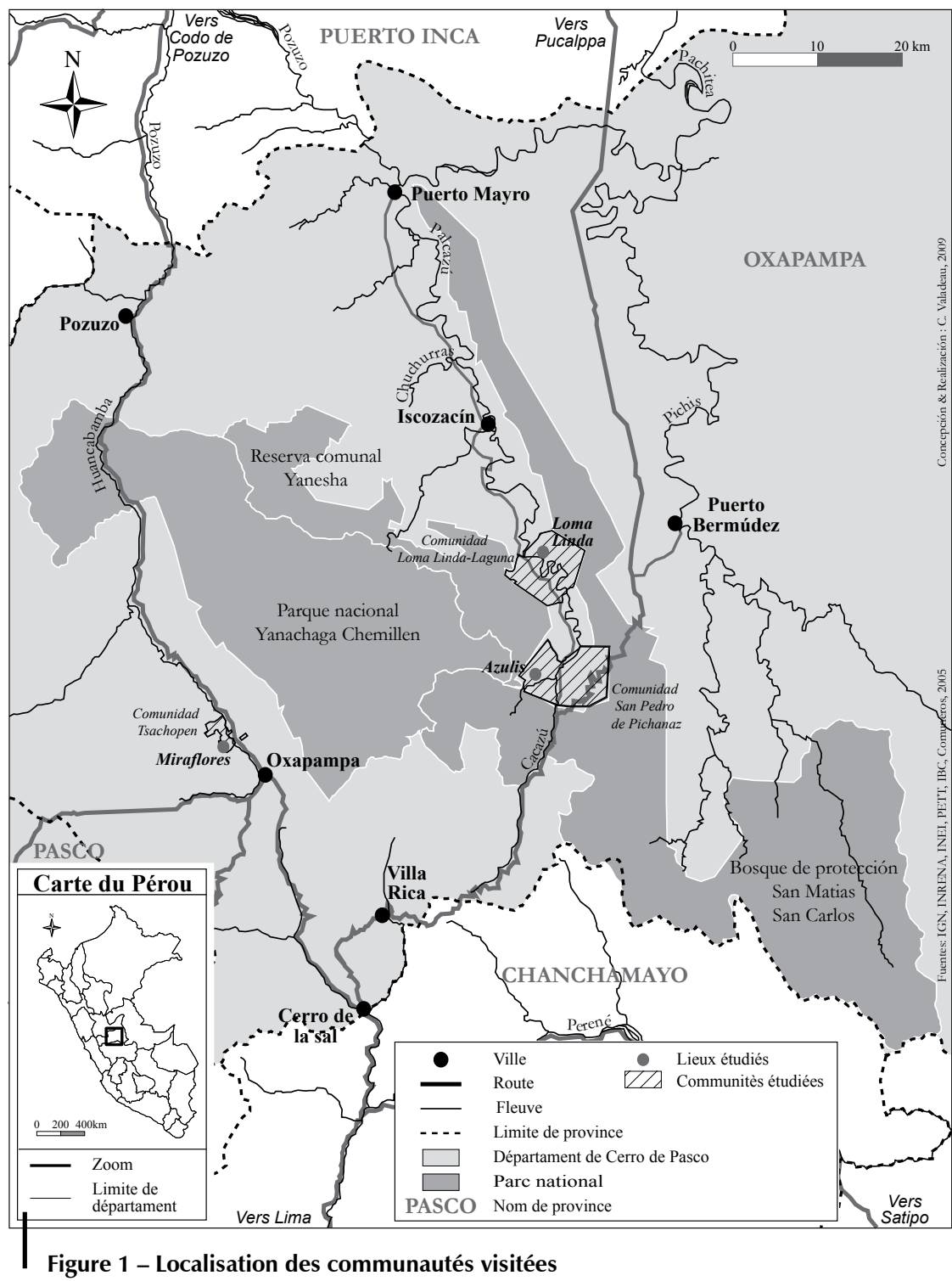

À l'origine, ce suffixe s'utilise dans le but de définir les différents groupes d'êtres mortels appartenant à arromñatenesha', I'humanité. Le suffixe -nesha' se lie à une unité lexicale dont la signification permet de préciser l'identité du groupe. II s'agit par exemple de Corneshamara'ynesha', les personnes faisant parties du groupe des « gens qui savent», Hua'ytanesha', « ceux qui savent chanter » ou encore Taraterenesha', « ceux qui viennent du martin pêcheur et qui savent pêcher ». À I'intérieur de chacun de ces groupes d'êtres il existe des familles ou des lignages, pour reprendre le terme employé par Marta Duff à cet égard (Duff Tripp, 1997). 
Lorsqu'il est question de famille, I'unité lexicale s'appose avec le suffixe -na'tar. $C^{\prime}$ est ainsi qu'il est possible de définir chez les Yanesha des familles portant le nom de Carapenna'tar, « ceux de grande taille », Huacana'tar, « ceux de l'échassier » ou Choyeshe'matsna'tar, « ceux venant des ombres errantes». Au sein de chaque famille, les personnes partagent toutes les mêmes caractéristiques physiques et comportementales. C'est ainsi que Les personnes appartenantà Choyeshe'matsna'tar ont une peau claire et sont décrites comme possédant un caractère souvent mal intentionné. Au sein de chacune de ces familles, il existe un géniteur. Afin de le désigner, le suffixe employé est -na'. Plus largement, il peut aussi être utilisé pour désigner un membre isolé de la famille. Huana'rrona' est donc le géniteur de la famille des Huana'rrona'tar appartenant au groupe des Taraterenesha' où se situe également la famille Huacana'tar. Ce sont des personnes connues comme possédant la qualité de savoir bien pêcher et qui vivent généralement proche des rivières. Le géniteur est décrit comme la personne qui ontologiquement a donné la lignée, il prit sa forme actuelle au moment de la spéciation. Enfin, chaque personne appartenant à une famille possède un nom commun, aujourd'hui en espagnol afin d'être référencé dans les registres administratifs. Toutefois, chacun possède un nom commun Yanesha utilisé couramment lorsqu'il est enfant. Ce nom est donné à l'enfant dès que celui-ci manifeste un trait de caractère précis, en d'autres termes que celui-ci présente une ébauche d'humanité. La construction de I'humanité d'un être se fait au moyen de divers végétaux permettant l'acquisition progressive des traits comportementaux communs à tout être et spécifiques en fonction du lignage auquel l'enfant appartient. Outre les autres composantes de la personne tels yechoyeshe'm, l'ombre, perturbatrice de l'activité consciente, yen'ar et chetsots, deux termes désignant l'ensemble du corps, masse inanimée, et la tunique traditionnelle, marqueur identitaire, il existe Yecamquëm, le principe vital qui donne vie au corps grâce à ses propriétés d'adhésion et de diffusion, et confère à l'être son intentionnalité. Yecamquëm est aussi garant de l'humanité de la personne.

Les plantes utilisées par les Yanesha dans le cadre de la construction de l'être humain ou plus largement dans le système nosologique global, apparurent dans les temps primordiaux, lors des évènements de spéciations qui débutèrent avec les colères de la Divinité Yompor Ror (Notre Père le soleil), frère jumeau de Yachor Arror (Notre Mère la lune) et leur ascension dans le ciel. Cette ascension signe le début de l'apparition de toutes les espèces présentes aujourd'hui sur terre. Cette période de spéciation prit fin lorsque toutes les Divinités eurent rejoint leur lieu de résidence définitif. Depuis ces évènements, les espèces douées de croissance autonome, telles les plantes, ont un corps désigné par le terme puechor, terme signifiant plus précisément « qui possède des branches ». Ainsi, à l'instar de tous les êtres vivants, tous les végétaux possèdent yechoyeshe'm, une ombre projetée au sol et yecamquëm, un principe vital végétal leur conférant intentionnalité et agentivité (Valadeau, 2010). L'ensemble du règne végétal ne se définit pas par un terme exact qui lui serait totalement englobant. On distingue des grandes catégories d'usage de plantes, et par extension, les plantes qui servent à cet usage prennent ce nom générique global, se rapportant à l'usage auquel elles sont 
destinées. Ainsi, les plantes dites « utiles», sherbets, telles que celles qui vont être mises à contribution lors des constructions de maisons ou pour la confection d'objets de la vie quotidienne, se distinguent des plantes alimentaires, ame'taña, et des plantes dites « médicinales», ou « magiques» appelées pare'shemats. Ces catégorisations d'usage ne sont pas immuables et excluantes, étant donné qu'une plante peut être utilisée dans différents buts. Plusieurs de ces termes peuvent être indifféremment et successivement utilisés pour parler d'une espèce. Cette étude s'intéresse aux pare'shemats. Étymologiquement, pareteñets signifie «se soigner avec des végétaux » et shemateñets " éclairé par le soleil de Yompor ». Utiliser un pare'shemats pourrait donc se définir par l'acte de se soigner avec des végétaux qui ont reçu la lumière/l'énergie/le principe vital de Yompor Ror, Notre Père le soleil. Les pare'shemats sont des plantes qui interagissent avec I'humain, autant de manière quantitative que qualitative, dans l'épreuve de la « maladie » et lors des constructions identitaires afin de donner ou d'ôter du principe vital. Le concept de pare'shemats se rapproche ainsi de celui de rao des Shipibo (Tournon, 2006 ; Rama Leclerc, 2003). Le rôle de ces plantes, qui dépasse largement celui des plantes plus communément appelées " médicinales», est d'agir sur le yecamquër $\tilde{m}$ des humains, de permettre ainsi la reconstruction, le maintien ou la construction d'une personne perçue incomplète à un moment précis de son existence, qu'elle soit malade, vieillissante ou encore non totalement humaine. En ce qui concerne la «maladie », elle est conçue essentiellement telle " une expérience de la métamorphose »(Taylor, 1997). La personne se rapproche alors d'un état transitoire entre l'humain et le non-humain, où deux issues sont possibles : I'humanité ou la non-humanité. Les Yanesha définissent deux sortes de « maladie » ou état dégradé de santé : atsnañets, les maux provoquées par des entités étiologiques et tsamneñets, les actes de sorcellerie ou plus largement de mauvaises pensées exprimées engendrant des douleurs (Smith, 1977 ; SantosGranero et al., 2004 ; Valadeau, 2010).

Par ailleurs, les travaux portant sur les catégorisations vernaculaires des végétaux furent nombreux. Brent Berlin aborda les systèmes taxonomiques mésoaméricains grâce à une approche ethnolinguistique et proposa un cadre universaliste de leurs études, celui des folk taxonomies1 (Berlin, 1992). D'autres recherches, prirent des chemins d'analyse différents en observant les rapports que les Hommes partageaient avec leur environnement. Cet article propose de décrire tout d'abord l'univers de certaines entités étiologiques du monde " non-visible » puis une partie de celui des plantes pare'shemats permettant de remédier au mal qu'elles peuvent induire (atsnañets). Les végétaux utilisés afin de pallier aux maux induits par des actes de sorcellerie ou de certains animaux et insectes appartenant au monde «visible » ne sont pas traités dans ce document. Cette analyse s'appuie sur les mythes recueillis lors des enquêtes ethnographiques et sur les données fournies par l'étude de la nomenclature des plantes Yanesha. Enfin, elle s'intéresse

1 Ce principe taxonomique se présente telle une arborescence de cinq rangs (taxon initial, forme du vivant, niveau générique, niveau spécifique et niveau variétal). Ce schéma d'organisation se base sur l'aspect morphologique des espèces définissant ainsi des niveaux classificatoires. 
aux logiques qui interviennent lors du choix thérapeutique, au moment où le diagnostic désigne une entité étiologique précise.

\section{LE MONDE ÉTIOLOGIQUE}

Les causalités ou étiologies des «maladies » se comprennent à travers les narrations des évènements qui eurent lieu dans les temps primordiaux. À l'identique de ceux d'autres sociétés amazoniennes, comme celles des Yuracaré (Hirtzel, 2010) ou des Achuar (Descola, 1986) par exemple, les mythes décrivent la période de spéciation des êtres de l'univers terrestre comme une époque durant laquelle certains hommes se sont différenciés des autres en prenant un corps différent, celui des êtres des différents règnes (Lévi-Strauss \& Eribon, 1988)2 :

« [...] Avant cette ascension (de Yompor Ror, Notre Père le soleil et de Yachor Arror, Notre Mère la lune), les animaux, les plantes, les minéraux, les esprits mellañoteñ, les démons, les hommes et les Divinités avaient tous forme humaine et vivaient ensemble. II n'existait alors ni la maladie ni la mort. À cette époque, ils (certains êtres) perdirent leur forme humaine et se distinguèrent physiquement des humains. De même, le territoire jusqu'alors commun se divisa. Les esprits mineurs de la montagne appelés mellañoteñ et les démons partirent vivre dans les lacs, les grottes et les rivières. [...]»

L'apparition de ces espèces signe également la division de l'univers terrestre en entpo', c'est-à-dire dans le «monde de ce qui se voit», le «visible » où vivent les mortels et celui du «non visible » dans lequel résident les êtres immortels, ama entopahuo, « ce qui ne se voit pas». À partir de ce moment, la mort et la « maladie » existent dans le monde du « visible » qui en était jusqu'alors exempt. Dans ce monde « visible», certains êtres ont gardé leur forme humaine originelle. Ils forment aujourd'hui arromñatenesha', I'humanité. D'autres se transformèrent ou furent transformés en mammifères, poissons, oiseaux, plantes ou encore en roches ou monts. Depuis ces évènements, les espèces douées de mouvement, les animaux, se reconnaissent à travers des corporéités certes distinctes, mais désignées par l'unique terme pue'moquër exprimant le corps en mouvement d'un être non-humain. Les autres espèces douées de croissance autonome telles les plantes, ont aussi un corps désigné par le terme puechor. À l'instar des humains, toutes ces espèces possèdent un yecamquëm, un principe vital leur conférant intentionnalité et agentivité, et un yechoyeshe'm, une ombre. Les entités bienfaisantes ou maléfiques, quant à elles, prirent place dans le monde

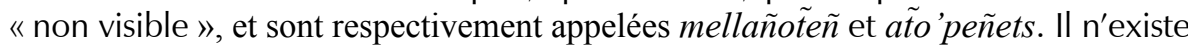
toutefois pas de frontière rigide séparant ces deux mondes et qui confinerait chaque être dans un univers. Certaines entités étant capables de se matérialiser dans le monde « visible », elles peuvent donc passer d'un monde à l'autre. La

2 Le mythe pourrait se définir par « une histoire du temps ou les hommes et les animaux n'étaient pas encore distincts » (Levi-Strauss \& Eribon, 1988). 
présence des êtres du monde «non visible » peut se constater grâce aux traces tangibles que ses habitants laissent parfois dans le monde « visible ».

Ontologiquement immortelles, les entités étiologiques appartiennent au monde « non visible », ama entopahuo. Elles possèdent toutes la caractéristique commune de ne pas être ordinairement visibles pour les humains. Deux classes d'êtres

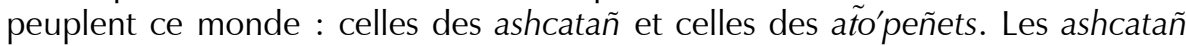
sont les Pères des animaux envoyés par les mellañoteñ gardiens des montagnes et créatures de Yato' Yos, Notre Grand-Père Yos, la divinité créatrice des hommes. Les at̃o 'peñets sont des créatures de Yosoper, frère malintentionné de Yato' Yos, et seront plus particulièrement traité dans cet article. Toutes ces entités étiologiques se plaisent à « heurter » le corps des humains, pocta'peñets, afin de dérober une partie ou la totalité de leur principe vital, sachyo'teñets, "soustraire le principe vital ». Cette soustraction laisse le plus souvent la personne dans un état d'inanition ou de semi-conscience auquel s'associe une symptomatique variable.

Dans le monde «non visible », les entités nommées a to ’peñets sont les fruits des réalisations de Yosoper, apparaissant à la même époque des temps primordiaux. Les at̃o'peñets se divisent ontologiquement à leur tour en deux groupes d'entités étiologiques: les tsapo'marnesha', " celles qui marchent la nuit », douées de facultés de matérialisation dans le monde "visible », et les yeto'marnesha', « celles qui marchent le jour à midi », responsables de certaines manifestations climatologiques. Au moment de la grande ascension des Divinités, ces entités restèrent sur terre, dans le monde " non visible », et ne perdirent pas leur immortalité. Aujourd'hui, elles y résident toujours, mais se sont isolées dans des lieux escarpés souvent difficiles d'accès aux humains. Créatures de Yosoper, ces entités maléfiques ne possèdent pas les mêmes composantes personnelles que celles des humains. Dans le monde tangible, elles ne possèdent pas de yeshoyeshe'm, car elles vivent soit la nuit, période où l'ombre n'est pas, soit la journée à midi, heure du zénith où l'ombre ne se voit pas. D'autre part, ces êtres de Yosoper possèdent le principe vital de Yosoper connu sous le nom de amachyorañ, et non pas celui de camuecñets, le souffle de vie de Yato' Yos. Ce souffle vital est un don de Yosoper à ses créatures, qui leur confère, au même titre que camuecñets, toute leur intentionnalité, volonté propre, conscience de soi et agentivité, dirigée le plus souvent à l'encontre des humains ou des autres créatures de Yato' Yos. Du point de vue de leurs corporéités, les a to 'peñets, entités invisibles pour le commun des mortels, apparaissent globalement comme des êtres humains. Mais elles possèdent certaines caractéristiques physiques, attributs corporels ou extensions du corps (telle pilosité excessive) et/ou de la cushma qui signalent définitivement leur non appartenance au monde des humains et/ou définissent aussi leur capacité de transformation : les ailes de chauves-souris de l'entité Oneñet, par exemple, renvoient à son pouvoir d'en prendre l'apparence. Le passage de ces entités dans le monde tangible se fait en prenant la corporalité pue'moquër, celle d'un corps en mouvement d'un être non humain : celle de mammifères, de poissons, d'insectes ou d'oiseaux.

Les tsapo 'marnesha' sont les entités étiologiques qui se transforment et marchent de nuit. Les entités étiologiques d'activité nocturne sont les plus nombreuses. À 
I'instar des animaux nocturnes, elles sortent à la tombée de la nuit au moment où les humains sont le plus vulnérables. Les tsapo 'marnesha' se distinguent tous par des corporéités différentes, et des lieux de résidence distincts. Leurs caractéristiques physiques, qui permettent d'identifier leurs pouvoirs intrinsèques de transformation, et par extension de passage dans le monde tangible, sont décrites dans les mythes et dans les représentations iconographiques. Les entités maléfiques de Yosoper résident pour la plupart d'entre elles dans « les hauts», endroits accidentés, sombres et difficiles d'accès par lesquels il est peu recommandé de passer dès la tombée de la nuit. Ces entités maléfiques se regroupent toutes sous le terme aspent. Apparue dans les temps primordiaux, Oneñet est décrite comme la plus puissante de toutes. Oneñẽ̃ est représentée comme une femme très velue, possédant seulement 4 doigts, des ailes de chauves-souris, posorr, et une queue de tatou, asho'sh, dont elle peut prendre la forme lorsqu'elle déambule dans le monde « visible ». À l'image des colibris qui accompagnent les chamanes, les serpents qui trônent en haut de son bâton sont dits être ses disciples ou ses messagers. Ils l'accompagnent en permanence. Ainsi, deux serpents venimeux cheminant ensemble à la tombée de la nuit dans des lieux humides escarpés signent la présence immédiate d'Oneñet̃. Le contact rapproché avec cette entité est généralement foudroyant. Un rapport plus éloigné est certes très grave, mais pas mortel, entraînant troubles de conscience, fortes fièvres, suivis de vomissements lorsque l'état dégradé de santé s'aggrave. Les entités les plus proches d'Oneñet en terme de pathogénicité sont Pante, Jo', Arrot̃ter et Ellechañ. Pante est un chasseur dont les ailes d'oiseau et la queue de singe signalent ses capacités de transformation animale. Il est également décrit comme un homme au corps à la texture de carton monté sur de très grandes jambes, présentant une attitude souvent ironique et moqueuse lorsqu'il se trouve en présence des humains. À l'instar d'Oneñet, Pante possède comme messager une chouette, pompore', ainsi qu'un tapir qu'il chevauche lorsqu'il chasse avec ses flèches. Ainsi, la présence d'une chouette à côté de traces de tapir dénote la présence invisible et dangereuse de Pante dont le contact physique peut provoquer des fièvres suivies de vomissements et de fortes diarrhées. Jo' est une entité ressemblant à un petit homme ayant la caractéristique physique d'avoir un nez en forme de trompe rappelant le rostre des certains grillons nocturnes de carapace duveteuse grise appelés tac. Jo' se déplace toujours à reculons, transporte toujours avec lui un fémur humain, et la poudre grise de cet os recouvre ses mains et son corps. Lors d'un contact avec Jo', cette poudre provoque une forte asthénie rapidement suivie de vomissements. Par temps de pluie, Jo' cherche souvent à s'abriter sous les maisons, ce qui oblige à la construction de palissades pour éviter qu'il ne prenne l'habitude de venir s'y réfugier.

Une autre entité étiologique, connue pour vivre dans les hauteurs du mont San Matías, se dénomme Arrotter. C'est un petit homme possédant des ailes d'oiseau, qui a la faculté de se transformer en tarater, un martin pêcheur. Lorsqu'il n'est pas dans le monde « visible », il joue de la flûte en soufflant par son genou dans I'os de son tibia. Son chant est dit effrayant. Quelquefois, ce chant s'entend d'un mont puis d'un autre, car Arrotter possède la capacité de se déplacer très vite dans les airs. Toutefois, gêné par son tibia droit dont les trous empêchent une 
bonne articulation, il peut difficilement se mouvoir dans les montées. C'est cette faiblesse, souvent décrite, qui permet de l'éviter ou de s'enfuir à sa venue :

«[...] Personne ne put se sauver. Arroțer furieux, commença à briser le cou de chaque personne afin de tuer tout le monde. Une seule personne qui n'avait pas entendu son chant, s'échappa en courant rapidement dans une montée. Car il est dit que s'il peut courir rapidement grâce à ses grandes jambes, son genou l'empêche de gravir des montées trop raides. [...]»

Les symptômes engendrés lors de la rencontre avec Arrotter sont des maux de tête, une forte asthénie suivis d'éventuelles diarrhées pouvant conduire à la mort. Ellechañ est un petit homme trapu ayant une queue et les mains

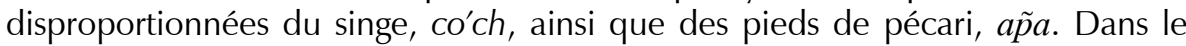

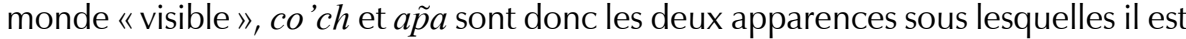
possible de voir cette entité. Si Ellechañ ne possède pas de messager, il est possible d'anticiper sa présence. En effet, il dégage une odeur caractéristique de viande en décomposition ou de couleuvre selon les descriptions. Un heurt avec Ellechañ est, là aussi, considéré comme très grave, la personne souffre généralement de fortes céphalées anticipant d'éventuels vomissements. Ces entités étiologiques nocturnes sont considérées comme les plus dangereuses pour l'être humain. Souvent causes de décès, les symptômes qu'elles entraînent après une rencontre oscillent entre fortes fièvres, maux de têtes, asthénie prolongée, puis d'éventuels vomissements. Actuellement, certains assurent que ces entités, terrorisées par le bruit des moteurs de voiture, se sont éloignées vers d'autres monts plus escarpés. Cela se remarque par le fait que les animaux témoins de leur existence se font plus rares, démontrant également leur éloignement. Mais elles se risquent cependant toujours à venir de temps en temps rôder près des habitations lorsqu'elles sont en chasse de yecamquërn.

À l'instar des tsapo'marnesha des hauteurs des montagnes d'autres entités dont les plus citées sont Arroshena', Alloch et Muentac, vivent aux abords des chemins accidentés qui montent des vallées vers les cimes, sentiers souvent empruntés par les humains pour accéder aux abattis. Ils sont dits aspentẽnesha'. Les Arroshena' sont semblables à des grands chiens sauvages dont la très longue queue ressemble à une fleur de roseau balayant l'air horizontalement. Le contact de leur queue avec le corps de quelqu'un entraîne de forts maux de tête et de violents vertiges accompagnés ensuite de vomissements. Les Arroshena' sortent dès la tombée de la nuit lorsque le temps est nuageux. Dans les temps mythiques, dans la région des montagnes du Yanachaga Chemillén, les Arroshena' étaient très nombreux mais suite à la vengeance d'un homme, seule une Arroshena' enceinte réussit à garder la vie sauve. Cela leur a permis d'exister encore et de peupler les monts aux alentours d'Oxapampa, tout en ayant une vraie peur des hommes. Dans les temps anciens, on dit que les Arroshena' vivaient dans des grandes grottes.

« Ils avaient la forme de grands chiens dont la queue rappelait la fleur des roseaux. [...] Les Arroshena' déjà saouls se servirent de la bière de manioc, puis mangèrent la pâte même à pleine main. Au petit matin, ils partirent, mais laissèrent toutes les saletés derrière eux, il y avait de la bière 
de manioc de tous les côtés. [...] En plein milieu de la nuit, dix personnes se rendirent à la grotte des Arroshena' et firent prendre un feu à l'entrée de la grotte, en y faisant brûler du piment. La fumée entra dans la grotte. Les Arroshena' se levèrent en toussant et dirent : " À cause de toi Grand-Mère, à cause de toi nous sommes tous en train de mourir ". Et tous les Arroshena' moururent, sauf une Arroshena' qui était enceinte et qui dormait dehors. Elle s'échappa en disant: " Je n'ai pas bu de bière de manioc, laissez-moi tranquille, ne me faites rien. "»

Alloch, lui, est une entité flottante dans les airs dont le pouvoir de transformation est a priori inexistant. En effet, les discours relatent que seuls les chamans ou les personnes consommant de la coca sont capables de le voir. Les autres décèlent seulement sa présence car il se dégage sur son passage une odeur âcre rappelant la viande avariée ou le chien sale. Le contact avec Alloch résulte en de fortes céphalées accompagnées ensuite d'éventuelles diarrhées voire d'hémorragies nasales. Vivant dans les monts de la région de San Pedro de Pichanaz, Muentac a l'aspect d'un petit homme n'ayant qu'un seul pied. II est d'ailleurs possible de remarquer le matin des traces de pas dont celle du pied gauche est l'empreinte circulaire de son pilon tibial. Muentac est décrit comme un bon chasseur capable de repérer ses proies de très loin. Son odorat très développé compense sa mobilité réduite. Il lui permet de se poster en attendant ses victimes dont il accroche ensuite les dents autour de son cou comme un collier. Lorsqu'une personne est la proie de Muentac, les premiers symptômes dont elle souffre sont des nausées et des maux de tête éventuellement suivis de vomissements. Hormis Alloch, les entités étiologiques qui vivent aux abords des chemins sont dites moins dangereuses qu'avant. Elles provoquent toutefois des états pathologiques sérieux dont les maux de tête, les nausées et d'éventuelles diarrhées ou les hémorragies nasales sont les principaux symptômes.

D'autre part, les entités étiologiques principales dont l'habitat se trouve à proximité des zones humides telles que les fossés, les ruisseaux ou les trous d'eau sont nommées росоуз. II en existe deux principalement connues; Rrotseñ déambule sur les berges des rivières pendant la nuit, et Mareñets près des puits d'eau à la tombée de la nuit. Rrotseñ est une entité se déplaçant très vite, car flottant dans les airs. Son corps est celui d'un squelette possédant un cœur et pourvu d'ailes d'oiseau blanches. Dans le monde « visible», son apparence est celle d'un oiseau nocturne appelé Rrotseñ qui sort à la tombée de la nuit. Tout l'être de Rrotseñ est sanguinolent. Ainsi, les traces de sang retrouvées sur les berges le matin à l'aube témoignent de son passage dans la nuit :

«Du temps de mon grand-père, il y avait beaucoup plus de démons qu'aujourd'hui. Les Rrotseñ suivaient souvent les gens lorsqu'ils passaient dans les fossés qui descendent de la montagne. Lorsqu'ils les attrapaient, ils leur mettaient de la terre dans les yeux, dans le nez et dans tous les autres

3 Pocoy dénomme aussi des êtres subaquatiques féminins. Comme les sirènes, leurs chants envoutent les pêcheurs dans le but de les entraîner dans l'eau (Santos-Granero, 2006). 
orifices du corps. Un jour, mon grand-père vit un Rrotseñ qui le suivait. II se mit dans un arbre, tout en haut afin que celui-ci ne puisse pas l'atteindre et attendit le petit matin pour descendre. »

Les symptômes que provoque cette entité sont généralement des gonflements corporels non douloureux, des fatigues, des fièvres, et dans les états jugés sévères, des hémorragies nasales. Mareñets, petit homme solitaire avide d'amour se promène sans arrêt avec un énorme fardeau sur le dos contenant toutes ses affaires ${ }^{4}$. Il se matérialise dans le monde « visible » sous la forme d'une petite mouche dont la bosse dorsale rappelle la charge qu'il transporte. Solitaire et toujours en quête d'amour, il se manifeste aux humains en leur provoquant des plaies suppurantes, douloureuses et d'aspect caractéristique. Si la personne atteinte ne soigne pas sa plaie, cela signifie qu'elle accepte cet amour. Une fois morte, elle rejoindra Mareñets. Toutes ces entités étiologiques du monde «non visible » sont douées de capacité de matérialisation dans le monde «visible ». Dans ce monde, elles prennent une corporéité animale qui est déjà pré-signifiée par les descriptions mihumaines/mi-animales qui en sont faites dans le monde «non visible ». Si certaines entités sont plus dangereuses que d'autres par l'intensité des symptômes évoqués qui peuvent rapidement conduire à la mort, elles provoquent toutes, peu ou prou, le même cadre symptomatologique peu spécifique (fièvres, vomissements, céphalées intenses, diarrhées, hémorragies). La pathogénicité mise en œuvre se résume toujours à une soustraction, voire un arrachement du yecamquëm de la victime. Ici, identifier la causalité du mal ne peut donc se faire sur la base de I'action pathogène induite. De plus, êtres de la nuit, ces entités, même dans le monde "visible », sont difficilement identifiables. C'est donc uniquement en se basant sur la connaissance de leurs lieux de vie, la présence de certaines traces laissées au sol ou l'apparition furtive d'un animal qui leur est associé, que des pistes peuvent être proposées. Cela permet de suspecter l'atteinte de telle ou telle entité.

Pour leur part, les yet̃o 'marnesha' ne possèdent pas de facultés de transformation, et ne possèdent pas d'attribut physique particulier. Êtres toujours invisibles, leurs caractères monstrueux ne peuvent être appréciés que par des personnes telles que les hommes mâchant de la coca ou des piri piri (Cyperaceae) spécifiques et le pa'llerr, le chaman. Ces entités signent leur présence dans le monde « visible » par des incidents climatiques. Contrairement aux entités étiologiques qui se déplacent la nuit, les yet̃o'marnesha' vivent — ont un état d'éveil — la journée. Elles ne sont pas dotées de capacité de matérialisation en être tangible. Elles restent donc invisibles aux humains. Leurs caractéristiques physiques rappellent les dommages qu'elles sont en mesure de provoquer chez les humains. Leurs conditions de déplacement ou de sortie sont liées à la présence d'éléments naturels (lumière, eau, vent, mouvements d'air) qui se combinent pour former certains évènements météorologiques, témoins de leur présence.

4 Les symptômes engendrés par Mareñets s'apparentent à ceux de la leishmaniose, maladie chronique parasitaire. 
Les entités étiologiques qui cheminent sous un temps ensoleillé ou quelque peu pluvieux sont principalement les Divinités cherepmarnesha' telles que Ayona p̃norrs et Cherop, ainsi que des mapuenesha' comme Huacash. Appartenant à la même famille que l'arc-en-ciel, elles entraînent toutes des éruptions cutanées et des prurits. Le pouvoir pathogène de l'arc-en-ciel est mentionné dans plusieurs sociétés amazoniennes. Glenn Shepard fait remarquer que I'apparition de I'arc-en-ciel signe la présence d'entités immatérielles (Shepard, 1999)6. Dans les temps primordiaux, Ayonap̃norr fut envoyée sur terre par les Divinités au moyen d'un arc-en-ciel, afin d'enseigner aux femmes Yanesha à cuisiner et à cultiver. Sa rapidité lui permettait de changer souvent de cushma, toutes très colorées et toujours différentes :

« [...] Un jour où sa cousine était particulièrement jalouse, elle déposa sur les tuniques d'Ayonap̃norr de la poudre de tsespan, une plante urticante. Lorsqu' Ayonap̃norr se changea, elle commença aussitôt à se gratter. Peu de temps après, elle eût mal. Voyant sa cousine en train de la regarder sans rien dire, elle lui demanda : "Cousine, pourquoi me fais tu cela ? Que t'aije fait ? Tu es bien méchante, Dieu m'a envoyée sur cette terre afin de vous aider à cuisiner vite et bien. Puisqu'il en est ainsi, je préfère m'en aller. " » Ayonap̃norr et son époux saluèrent, et décidèrent ensemble de quitter la terre. Aujourd'hui, il est dit que lorsqu'apparaît l'arc-en-ciel, ils sont en train de manger en nous regardant. »

Suite à ces évènements, Ayonap̃norr passa dans le monde « non visible » où elle se promène et mange de temps en temps sur les chemins. Depuis ce jour, il est dit que lorsqu'apparaît un arc-en-ciel, il est dangereux de se trouver sur son chemin car sa cushma est toujours irritante. Vivant à Tsachopen dans le secteur de Gramazú, Huacash s'est établi près d'un rocher en forme de taureau allongé. Selon les récits, sa présence se manifeste par de petits arcs-en-ciel en suspension près des puits d'eaux. Si les éruptions cutanées qu'entraîne une rencontre avec Ayonap̃norr peuvent se soigner, celles qu'engendre Huacash sont dites beaucoup plus graves, voire mortelles. Elles ont entraîné la désertion de lieux. Les maisons bordant le chemin menant à ce rocher ont toutes été abandonnées :

« Tous ceux qui se sont installés ici, soit moururent, soit s'en allèrent gravement malades $»$.

Enfin, Cherop, nom désignant à la fois la brume fine dans laquelle se difracte la lumière et une espèce d'insecte des milieux humides appelé ya'shenesha', est une entité des endroits humides et des sous-bois, qui provoque tout comme Huacash et Ayonap̃nor des cherpare 'teñets, rashs cutanés recouvrant tout le corps. Elle manifeste sa présence par des phénomènes de diffraction de la lumière. Les « maladies de l'arc-en-ciel » entraînent toutes en termes de symptomatiques

5 Divinité Yo'ch Ayonap̃norr, Notre Sœur l'arc-en-ciel (Smith, 1977 ; Santos-Granero, 2004).

6 Glenn Shepard compare l'arc-en-ciel à des vapeurs pathogènes, illness vapors. 
des rashs et des éruptions cutanés parfois accompagnés de fièvres et de grandes fatigues. Dans les Andes équatoriennes, Eduardo Estrella mentionne que I'arcen-ciel est dit provoquer le même type de symptômes (Estrella, 1981). Chez les Matsigenka, Glenn Shepard cite les nausées comme conséquences du contact avec ce phénomène climatique (Shepard, 2004).

Les mouvements d'air brutaux, ñorrareñets, formant des tourbillons soudains, signalent aussi la présence d'entités étiologiques nommées m̃orranesha'7. Paryacoñch et Aptallesha' sont deux agents pathogènes provoquant lors de leur passage des mouvements d'air évoquant de petits tourbillons soulevant la poussière et les feuilles mortes, morrareñets. Paryacoñch et Aptallesha' sont décrites comme parentes de la Divinité Huomenquësha', envoyée sur terre dans les temps mythiques afin d'engendrer des tempêtes et de troubler incessamment la vie des Yanesha. Paryacoñch est une entité se déplaçant dans les airs. Elle se plaît à déambuler près des habitations, cherchant à entrer en contact avec les humains. Aptallesha' est une entité dont l'habitat privilégié se situe sous les pacay (Inga sp.) qui bordent les plages des rivières. Les mouvements d'air tourbillonnant à l'approche de ces arbres dénotent sa présence, de même que des bruits insolites comme ceux que font les troncs quand ils tombent. L'action de toutes ces entités provoque un sévère état d'asthénie et des états nauséeux où « la tête tourne ». Cette symptomatique peut précéder des vomissements lorsque l'état déjà dégradé de santé se détériore.

Les abords des rivières sont peu conseillés à partir du moment où le soleil disparaît et qu'il ne frappe plus la surface de l'eau. Les principales entités déambulant sur les berges ou présentes dans les trous d'eau se nomment oñenesha', elles sont respectivement Omarñets et Sapeñets. L'entité étiologique Omarñets, le « noyé », ne se montre jamais dans le monde du « visible », mais il est toutefois possible d'entendre le bruit de l'eau qui remplit exagérément son ventre, et qui ressort de sa bouche à chacun de ses pas. Omarñets fait disparaître les enfants sous l'eau, relâchant les corps gonflés et tâchés d'ecchymoses. Une personne adulte contracte généralement des troubles cutanés. Vivant dans les ruisseaux d'écoulement des eaux vers les rivières, Sapeñets est une entité qui se plaît à consommer les principes vitaux des fœtus in utero. Bien que toujours invisible, elle se met à pleurer et à crier comme un nouveau-né après avoir pris son repas. Sapeñets peut être pathogène pour les femmes enceintes. Dès lors, pour celles-ci, une rencontre avec cette entité engendre de fortes douleurs abdominales pouvant conduire à des hémorragies utérines.

Enfin, les entités « de passage » sont de forme humaine. Au nombre de quatre, elles vagabondent ensemble à travers la vallée. Ce sont des affections qui sont envoyées épisodiquement par Yosoper. Contrairement aux précédentes, elles ne vivent pas tout le temps sur la terre. Une fois leur but destructeur atteint, elles regagnent leur royaume.

«[...] Créées par Yosoper dans le but de détruire le peuple Yanesha, 
Errasañats, Yonnañets, Shorañets, Puertsañats sont respectivement les incarnations de la fièvre hémorragique (fièvre jaune), de forte fièvre (paludisme), de rashs cutanés (variole) et d'affections cutanées formant des plaques rouges indurées (rougeole). Les maladies arrivèrent ensemble en haut d'un mont dans la région de Cacazú. Ils s'en allèrent descendant la vallée en direction d'Iscozacín. En chemin, elles tuaient toutes les personnes qu'elles croisaient afin de les manger. Elles se plaisaient à aspirer leur graisse en emportant toujours un petit peu dans leur sac afin de la consommer en chemin. Une fois en bas de la vallée, elles décidèrent de faire demi-tour, car elles constatèrent qu'il n'y avait plus personne à tuer. [...] ».

Maladies contagieuses et épidémiques apparues à l'époque de la conquête espagnole, elles firent beaucoup de victimes parmi la population Yanesha. Elles sont regroupées sous le terme atsnañets ${ }^{8}$. Reflet de cette époque coloniale, ces entités sont décrites à l'image de grands hommes blancs dont la pilosité est importante. Selon le mythe des entités « de passage » lors de leur première venue sur terre, elles arrivèrent dans les hauteurs de Cacazú et voyagèrent jusqu'au fond de la vallée de Palcazú. À l'image d'épidémies, elles se déplacèrent de lieu en lieu, d'habitation en habitation, tuant des centaines de personnes sur leur passage. Du point de vue de leur corporéité, chacune d'entre elles possède les caractéristiques physiques des symptômes dont elle est la conséquence. Errasañats possède en guise de cushma des ruissellements de sang inépuisables rappelant les hémorragies abondantes. De même, Yonnañets grelotte de froid à l'image des sensations qui peuvent être éprouvées lors des fortes fièvres palustres. Enfin, Shorañets et Puertsañats ont respectivement le corps couvert de boutons ou de pustules rouges rappelant les éruptions et rash cutanés typique de cette affection. Ces quatre voyageurs ont un appétit insatiable qui les pousse «à manger la graisse» de tous les humains se trouvant sur leur passage. Seule la présence avérée de la «maladie » signe la présence de ces entités.

En conclusion, toutes ces entités étiologiques invisibles trahissent, d'une manière ou d'une autre, leur présence dans le monde «visible ». Les entités nocturnes se matérialisent dans le monde « visible » ou y inscrivent des traces déchiffrables. Les entités diurnes ont une probabilité de présence plus élevée lors de certaines manifestations climatiques. L'action de certaines entités est signée par les marques corporelles qu'elles laissent sur le corps de leur victime. La connaissance de ces modes d'être dans le monde «visible », ou de ces caractéristiques comportementales, donne autant d'indications qui permettent de se prémunir, et d'anticiper par évitement, toute rencontre déplaisante. Il est aussi possible de soulever le point selon lequel, les trous d'eau, certaines des formations géologiques ou des évènements climatiques ne sont pas pathogènes en soi, ils représentent des lieux de vie auxquels sont associés certaines entités étiologiques. De part la potentielle présence de ces entités, ils sont donc potentiellement dangereux. Tout

8 Ce terme désigne les maux provoqués par une entité étiologique. Il fut probablement donné à ces entités de passage lors de l'arrivée des épidémies à l'époque coloniale. 
contact avec une de ces entités s'accompagne d'une atteinte du yecamquërn pour la victime, entraînant un état global de santé dégradé dont les symptômes associés sont variables : le cadre sémiologique des «maladies », les symptômes déclarés ne sont en rien spécifiques aux entités. En d'autres termes : à un mal ou à sa causalité ne correspond pas un syndrome ou un symptôme défini. II existe, à cet égard, une grande variabilité et imprécision descriptive dans les discours. Corollaire de cette affirmation, il est donc impossible en se basant sur les symptômes évoqués, ou ressentis et exprimés comme tels, de connaître avec précision la cause de la «maladie ». Dès lors, les critères de discrimination de ces « maladies »permettant de comprendre les réflexions individuelles et diagnostiques ainsi que les choix thérapeutiques émanant de ces conclusions - porteront, en partie, sur les représentations de la manière d'être au monde "visible » de ces entités incroyablement similaires dans l'imaginaire collectif de villages parfois très éloignés.

\section{LES ORIGINES DES PLANTES PARE'SHEMATS}

Certains mythes relatent I'histoire de différents personnages décrits comme étant à l'origine de l'apparition des plantes sous leur forme actuelle. Les plantes apparurent sous leur forme actuelle sur la terre à l'époque de la spéciation. Sous leur forme actuelle, elles sont : soit les états transformés de certaines personnes des temps mythiques, qui furent (1) des Divinités ou (2) des ancêtres, soit des plantes qui furent données directement en l'état par (3) certaines Divinités ou par (4) des entités du monde «non visible » restées sur la terre, mellañoteñ ou passées sur terre, entités « de passage ».

Dans le cas des Divinités et des ancêtres, leur transformation en être au corps de plante (puechor) est, soit consécutive à la mort du corps humain de l'ancêtre ou de la divinité, soit issue d'une de leurs productions corporelles (comme le lait ou le sang) tombée au sol, et le reste de leur corps prend alors une autre apparence, telle celle d'oiseau et autres animaux, ou encore d'étoile. L'apparition d'une plante a toujours lieu à la suite d'évènements généralement dramatiques (trahisons, dangers imminents) ou de découvertes fortuites, et est toujours présentée comme délibérée, intentionnelle ou motivée par un but spécifique, celui d'aider les Yanesha dans la construction et le maintien d'un état de bonne santé.

Selon les données recueillies, et les dires de Richard Smith, il existe plusieurs grandes familles de divinités, qui se positionnent les unes par rapport aux autres selon certains niveaux hiérarchiques. Toutes ces familles sont ordonnées selon le même schéma généalogique, avec le niveau le plus élevé dénommé Yato' (Notre Grand-Père) qui se trouve au rang hiérarchique supérieur. C'est, par exemple, le rang où se positionne Yato' Yos, Notre Grand-Père Yato' Yos, Yato' Yemats, Notre Grand-Père Tabac, ou encore Yato' Ramuesh, Notre Grand-Père Ramuesh. Au rang intermédiaire dans chaque grande famille se trouvent Yompor (Notre Père) et Yachor (Notre Mère). En descendant ensuite l'arbre généalogique, Yemona'sheñ (Notre Frère) et Yo'c̈h (Notre sœur) occupent le rang inférieur de l'arbre généalogique (Smith, 1977). Il existe donc des personnages mythiques 
qui peuvent être considérés comme des divinités de rang supérieur (Yato' Yos et ses envoyés, Yosoper et ses envoyés), et des divinités se situant plus bas dans la généalogie, considérées comme étant de rang inférieur.

Il est possible de positionner certaines des Divinités et les plantes qui en sont issues le long de cet arbre de parenté. C'est ainsi qu'au rang intermédiaire, le lait de Santosaprecoya, fille de Notre Grand-Père Yato' Yos, va donner naissance à une plante, pomocpar (Anthurium croatii Madison) :

« [...] Avant de rejoindre son père, Yato' Yos, dans le ciel, elle fit tomber des gouttes de lait de son sein, et déclara : " Avant de m'en aller, je voudrais vous laisser un présent. Où tombera une goutte de lait poussera une plante. Sa tige pourra vous servir pour soigner les infections » expliqua Pomoc." $[\ldots] \gg$.

À ce même rang généalogique, la Divinité Coca, Yachor Mom, se trouve rattachée à cette famille de par son union avec un des fils de Yato' Yos dénommé Yompor Ror. La coca porte aujourd'hui le nom de COC' $^{\prime}$ (Erythroxylum coca Lam.) :

« [...] Yompor Ror, furieux des infidélités de sa femme et du manque de respect de Huar, démembra sa femme et jeta les morceaux de son corps de tous les côtés. [...] À chaque endroit où tomba une partie de son corps, poussa un plan de coca identique à celui que nous consommons aujourd'hui. $9[\ldots] \gg$.

Dans ces deux cas, la transformation en plante se fait à partir d'une sécrétion du corps de la Divinité ou à partir des morceaux de son corps.

Par contre, pour les plantes issues de Divinités de rang inferieur, c'est le corps entier de la Divinité qui se transforme : la petite fille de Yato' Yos et fille de Notre Mère, Yachor Santosaprecoya, se dénommait Yo'c̈h Huallapnarrenana'. Elle se transforma toute entière en une plante dénommée aujourd'hui huallpnarrë̈h (Munnozia hastifolia (Poepp.) H. Rob. \& Brettell) :

" La fille de Notre Père Yato' Yos, Notre Mère, Yachor Santosaprecoya, laissa sa petite fille sur Terre avant de monter au ciel. Elle s'appelait Yo'c̃h Huallapnarrenana', et continua de vivre avec les Yanesha pendant quelques temps après I'ascension de sa grand-mère. À l'époque où toutes les personnes se transformèrent, elle se transforma en plante. Cette plante est connue sous le nom de huallapnarren. »

Petit-fils de Yato' Yos et fils d'Ayots, I'enfant Poporrona' prit, quant à lui, I'apparence d'un palmier appelé aujourd'hui poporr (Bactris gasipaes Kunth)10.

« [...] Essayant d'abattre Poporrona', ils n'arrivèrent qu'à s'entretuer. Poporrona' leur suggéra de construire un radeau amarré au milieu de la rivière et de l'attacher dessus assis sur un siège. Une fois attaché, ils devraient lui enfoncer un pieu dans le crâne et revenir cinq jours plus tard.

9 Ce mythe est également relaté dans d'autres travaux (Smith, 1977 ; Santos-Granero, 2011).

10 Fernando Santos-Granero mentionne également ce mythe dans le contexte de description des enfants sorciers (Santos-Granero, 2002). 
Les gens suivirent ses instructions et le laissèrent mort sur le radeau. Le cinquième jour après son exécution avait poussé à la place de sa dépouille un palmier rempli de fruits. »

Les plantes peuvent aussi être issues d'ancêtres transformés. D'un point de vue ethnobotanique ce groupe de plantes représente la majorité des plantes collectées. Ce sont en général des espèces faciles à trouver, sylvestres ou croissant spontanément autour des habitations. Dans ce groupe de plantes une différence est faite entre les plantes issues d'ancêtres ayant eu des rapports étroits avec les Divinités, car ils les avaient épousées ou étaient leurs serviteurs, et les autres, sans relation particulière avec les êtres divins. Ces liens particuliers avec les Divinités (union ou bien service) positionnent de fait la personne dans un niveau social supérieur ou inférieur, et partant de là influencent sur le processus de transformation qui se fera sur la base d'une partie du corps ou de son intégralité, reproduisant le modèle mis en évidence au sein des êtres divins de la grande famille de Yato' Yos : la base de transformation du corps ontologique varie, et cela en fonction du rang social de l'ancêtre ayant donné naissance à la plante. C'est ainsi qu'est expliquée l'origine de la plante pa'yon (Tetragastris panamensis (Engl.) Kuntze) qui sert à teindre les tuniques d'ocre-rouge :

« Dans les temps primordiaux, Yompor Oreseñ était une Divinité qui vivait sur cette terre, et avait deux épouses qui s'appelaient Hueraresyac et Shopsheresyac. Un serviteur dénommé Pashtason vivait également avec eux, et les aidait aux tâches quotidiennes. [...] Un jour Pashtason coupa le sexe (clitoris) de Shopsheresyac, une des femmes qu'Oreseñ lui avait laissé en partant au ciel. Le sang qui tomba sur le sol à cet instant pris la forme d'un arbre, pa'yon [...]».

Shopsheresyac elle-même fut ensuite élevée au rang de Divinité, et s'éleva dans le ciel avec Huerarescyac, pour former des étoiles, qui vivent dans la voie lactée. Parmi les ancêtres Yanesha qui vouaient une immense dévotion aux Divinités et étaient à leur service, il y avait la servante de Santosaprecoya, qui se transforma en une plante connue aujourd'hui sous le nom añchechpar (Ossaea sp). Cette plante était la servante de Notre Mère Santosaprecoya :

« Lorsque Santosaprecoya partit rejoindre son père Yato' Yos en laissant une goutte de lait par terre, sa servante ne voulut pas rester seule sur terre, et décida de faire pareil et son corps prit la forme d'une plante. »

D'autres ancêtres se transformèrent en plante sans toutefois qu'il y ait de lien avec de quelconques Divinités. C'est le cas de la grand-mère Orroshe'sh et de ses deux petites-filles. Elles se transformèrent en plantes de orroshe'shpar (Dracontium peruvianum G.H. Zhu \& Croat, Dracontium plowmanii G.H. Zhu \& Croat et Dracontium spruceanum (Schott) G. H. Zhu :

« [...] Un jour où Orroshe'sh consomma plus de piment qu'à l'ordinaire, ses cheveux commencèrent à tomber. [...] Ses petites-filles lui répondirent : " Grand-mère, pourquoi as-tu mangé tant de piment ? Cela fait des jours que nous te disons de faire attention. Mais tu as l'air bien triste, viens donc dormir avec nous ". Ainsi, la nuit s'avançant, Orroshe'sh s'en alla dormir avec 
ses deux petites-filles. Au petit matin, lorsque sa fille revint à la maison, elle chercha sa mère et ses filles afin de les embrasser et d'annoncer sa venue. «Maman, maman ? Les filles ? Où êtes vous ? " Elle avait beau les appeler, personne ne répondait, alors elle se mit à chercher dans toute la maison, elle se rendit au bout d'un moment dans leur chambre. Là, elle ne vit personne, il y avait dans le lit une plante connue aujourd'hui sous le nom de orroshe'sh, Sacha Jergón ou encore Pituquillo del monte en espagnol. La grand-mère Orroshe'sh et ses deux petites-filles s'étaient transformées dans la nuit en plantes médicinales. Utilisées en bain de vapeur, elles sont connues pour traiter les piqûres de serpent. »

Au sein de chaque groupe de plantes provenant d'états transformés des ancêtres, ou de Divinités de rang intermédiaire ou inférieur, les hiérarchies ontologiques sont donc organisées selon un schéma reprenant un ordre généalogique ou social. II s'inscrit dans le corps physique ontologique de la plante en définissant la partie de l'être qui servira de base à la transformation (corps, sécrétion, partie de corps). La position généalogique ou sociale ontologique se projette donc dans ce qui servira de base à la transformation. Une organisation semblable est remarquée par Fernando Santos-Granero. Il catégorise les plantes cultivées en fonction leur mode de transformation ; grotesque ou sublime (Santos-Granero, 2011). La transformation lacto sensu semble donc permettre la catégorisation. À cette hiérarchisation se juxtaposent aussi des valeurs de puissance ontologique. Cela se traduit, après la transformation, en notion d'efficacité thérapeutique du remède : les espèces ontologiquement proches des Divinités seront considérées comme plus «puissantes », au contraire d'espèces n'ayant pas de lien particulier avec celles-ci.

D'autres plantes furent offertes directement aux humains sous leur forme végétale. Ces dons sont le fait de Divinités éprouvant amuereñets, de la compassion pour les Yanesha. Positionnées au sommet de l'arbre généalogique, ces plantes furent données directement ou indirectement à travers des personnages mythologiques élevés au rang de cornesha', représentant spirituel, ou d'entités du monde «non visible ». La plupart des plantes furent offertes par des cornesha' mythiques vouant leur culte à Yato' Yos, par la Divinité Yato' Yos lui-même et d'autres entités du monde «non visible » tels les mellañoteñ et les entités « de passage ». Même si l'origine ontologique de ces plantes ne les relie pas à l'humanité, elles sont considérées néanmoins comme imprégnées d'un yecamquëm de l'être qui en fit le don. Elles furent données directement par des Divinités de rang supérieur aux hommes, ou indirectement au travers des cornesha' leur vouant un culte (ex. Poramasa', le Père des vautour). Parmi les plantes données par les Divinités/ cornesha', se trouvent des espèces de haute valeur culturelle, souvent cultivées, et de grande importance dans la vie quotidienne : en particulier le tabac ou encore certains souchets (epe' en Yanesha), plantes données pour aider dans les tâches quotidiennes, améliorer les capacités intellectuelles et physiques :

«[...] " Je (Poramasa') t'ai ramené des herbes magiques, elles s'appellent

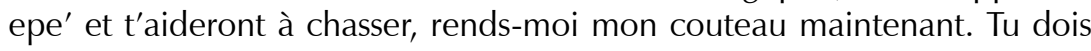


les planter à côté de chez toi, et le dire à ta famille et tes amis. Protège les biens. " C'est ainsi que le Père des vautours (Poramasa') 11 enseigna au chasseur comment utiliser les souchets afin de chasser plus facilement. L'homme, convaincu, lui rendit alors son couteau. »

De nombreuses plantes furent également l'objet de don de la part des êtres que créèrent respectivement Yato' Yos et Yosoper, les mellañoteñ et les entités " de passage », aujourd'hui retranchés dans le monde " non visible ». Matérialisant toujours un sentiment de compassion, ces dons sont aujourd'hui les plantes les plus communément utilisées. Les mellañõ̃en, sorte d'esprits protecteurs des lieux qui peuvent aussi châtier les Yanesha non respectueux en leur envoyant des entités étiologiques aux atteintes sévères, ont, pour leur part, donné toutes les plantes connues sous les noms génériques de campuerpan et corarnopan. À coté de ces plantes, dons des mellañõ̃eñ, se trouvent les dons d'Errasañats et de ses compagnons, entités pathogènes de Yosoper. Malgré son appartenance au monde maléfique Errasañats eut pitié des Yanesha. Avant son départ, il leur indiqua des plantes nommées errasañatspan.

Les plantes « données » ne subissent donc pas de transformation, mais se présentent toujours comme la matérialisation d'une intention/volonté émanée directement de Yato' Yos, de certains cornesha' mythiques, ou bien comme la matérialisation d'un sentiment de compassion énoncé à voix haute et motivé par une situation précise. Si certaines sont plus valorisées que d'autres (epe', ou yemats, le tabac) c'est parce qu'elles sont l'intention matérialisée de Divinités puissantes elles-mêmes. De ce fait elles peuvent être considérées comme des plantes civilisatrices, à l'origine de la fondation de la culture Yanesha. Les autres, dons des entités du monde « non visible », apparaissent comme de simples plantes médicinales capables de remédier aux états de dégradé de santé que peuvent causer ces mêmes entités aux Yanesha.

\section{LES COMPOSANTES DE LA PLANTE}

A priori, les plantes, telles qu'elles apparaissent aujourd'hui, sont toutes constituées d'un corps physique, puechor, et d'un yecamquëm, principe vital individualisé, qui peut interagir avec celui des humains. Cette interaction se fait grâce au remède, qui est à son tour l'état transformé d'une plante.

Les Divinités de rang intermédiaire et ancêtres de hiérarchie sociale supérieure ont pour base de transformation une partie de leur corps ou une production corporelle. Que cette production corporelle soit urine, sang ou lait, le remède pouvant être préparé avec la plante est en tout point semblable à son origine ontologique.

La plante pomocpar (Anthurium croatii Madison), par exemple, est l'état transformé d'une goutte de lait d'une Divinité tombée au sol. La racine de cette

11 Le vautour dit gallinazo en espagnol se détermine en systématique par Cathartes aura. 
plante préparée en décoction donne un remède «blanc crémeux semblable à du lait en poudre mélangé à de l'eau ». De même, l'écorce de l'arbre pa yon (Tetragastris panamensis (Engl.) Kuntze) longuement bouillie fournit un décocté rouge sang nommé tsasasen, liquide qui prend une couleur rouge. Il est utilisé dans les cas d'hémorragie féminine, qui s'apparente aux gouttes de sang perlant à cause de la blessure dont fut l'objet l'ancêtre de rang supérieur Shopsheresyac.

En ce qui concerne les plantes issues de Divinités de rang inferieur et les ancêtres « ordinaires », le lien avec leur origine ontologique se joue au niveau de l'aspect physique non transformé du ou d'une partie du végétal, rappelant une caractéristique physique ou comportementale de l'ancêtre, et qui se trouve être aussi la partie de plante employée dans le remède : épine du parépou, poporr (Bactris gasipaes Kunth)12 rappelant les flèches de ce dernier, ou tige résistante de la plante (Satyria panurensis (Benth. ex Meisn.) Hook. f. ex Nied.), qui souligne la force qui habitait l'ancêtre po'huamencarec̈hpar.

Ainsi, pour les plantes issues des productions corporelles des ancêtres de rang social supérieur ou de Divinité de rang intermédiaire, la théorie des semblables s'utilise en associant les caractéristiques du remède préparé à celles de la sécrétion originelle. Le remède peut donc être considéré comme une production d'un organe du corps actuel de la plante, et la préparation du remède comme une nouvelle transformation permettant de recréer la fonction physiologique ontologique. Dans les autres cas, c'est une des caractéristiques ontologiques (physique et comportementale) de l'être avant la spéciation qui, par analogie, sera un indicateur de la partie de plante à employer lors de l'élaboration du remède. Ainsi, la base de transformation de l'être ontologique est alors analogue à la forme que prend le remède, et la transformation eut lieu lors de l'énonciation de l'intentionnalité des personnages ontologiques ponctuant alors le moment de I'apparition de ces plantes sur terre.

\section{INTENTIONNALITÉ, PRINCIPE VITAL ET USAGE DES PARE'SHEMATS}

Dans les mythes, si les évènements qui entrainèrent l'apparition des plantes pare'shemats sont de nature différente, la cause première de la spéciation/ transformation semble toujours être une volonté individuelle.

Dans les cas où les plantes sont des états transformés de corps entiers ou de productions corporelles, qu'elles soient issues d'ancêtres ou de Divinités de rang inférieur avant de changer d'apparence, le personnage mythique énonce toujours à haute voix les raisons de son acte et la destinée de la plante à laquelle il est en train de donner naissance. C'est par ce discours que l'usage de chaque plante pare'shemats se définit :

«Dans les temps anciens, une femme donna naissance à un enfant au bout de seulement quatre mois, mais seules la tête et la figure de l'enfant étaient

12 Le parépou est appelé pijuayo en espagnol régional. 
formées, le reste n'était que du sang. Après l'enterrement, l'enfant mort-né, qui était une petite fille, apparut à sa mère et lui dit : "Maman, je suis ta fille, celle que tu enterras là-bas... Regarde-moi ! À la place du sang que je vais perdre sur le sol, il poussera maintenant des plantes de sapeñtsopar13 qui serviront contre les hémorragies des femmes " [...]».

L'énonciation, prélude de transformation, est donc directe, s'adresse à un être humain, et vise à conférer un usage précis à une plante. Elle peut aussi s'appliquer à un groupe de plusieurs plantes liées par des liens de parenté/relationnels/sociaux ontologiques particuliers. À ce moment, les êtres mythiques liés se transforment simultanément et les plantes qui en sont dérivées possèdent des usages communs. Dans d'autres cas, les circonstances de la transformation sont comme une mise en scène :

«Yachor Yaneshallem̃ était une femme qui vivait jadis sur la Terre. Un jour où les Yanesha s'aperçurent de sa réelle identité, elle déclara : "Je ne suis pas humaine, je fus envoyée par Notre Grand-Père afin de vous aider à toujours avoir de quoi manger. " Un homme lui demanda alors : "Mais que devons nous faire? "Yaneshallem répondit : " Maintenant vous devez aller dans vos abattis, là où les herbes sont hautes. "Les gens accompagnèrent Yaneshalleñ, elle se mit au centre de l'abattis et dit : "Vous devez tous faire tomber les arbres des quatre coins du champ sur moi, afin qu'ils me tuent... ". Des haricots sauvages poussèrent de chaque goutte de son sang tombé à terre. »

Par contre, dans les cas où les pare'shemats sont issus de dons provenant de Yato' Yos et/ou Yosoper (ou des êtres qui leur sont affiliés tels que cornesha' mythiques, mellañoteñ et atsnañets), cette énonciation verbale, ainsi que les circonstances détaillées de la spéciation ne sont jamais mentionnées dans les mythes. Tout se passe comme si ces plantes étaient des émanations de la volonté supérieure de ces personnages mythiques, ne nécessitant l'apport d'aucun support physique/ matériel pour s'incarner. Quoiqu'il en soit, volonté pure ou circonstances particulières motivant une transformation, cette intentionnalité est à la racine de la création du principe vital, le yecamquëm de la plante. À ce moment, le yecamquëm de la plante pare 'shemats possède une spécificité d'usage, qui sera mise à profit pour soigner l'être humain malade. Il existe donc une étroite correspondance entre l'intention d'apparition ontologique du végétal et son principe vital végétal actuel. C'est ce yecamquër̃ végétal, sorte de volonté ontologique matérialisée, qui interagit avec l'être humain et est à l'origine de l'usage thérapeutique des pare'shemats : autant d'intentions énonçant la volonté de créer une plante sont ainsi à l'origine d'autant de yecamquër̃ végétaux.

${ }^{13}$ Le terme sapeñets désigne à la fois le fotus et une entité étiologique provoquant de fortes hémorragies utérines. 


\section{COMMENT NOMMER LES PLANTES?}

Les espèces végétales peuvent être regroupées selon leur écotope : les plantes cultivées, narmets, se trouvent dans les abattis des femmes (chets), ou des hommes (ра'muer) et proche des zones entourant les habitations (pamp). Les plantes sylvestres sont dites $t a$ 'te, et viennent de zones de forêt secondaire, potsat̃, et de forêt inaccessible primaire, puerĩarer. Cette distinction entre les plantes cultivées et les plantes sylvestres est également décrite chez les Yagua par Jean-Pierre et Bonnie Chaumeil (Chaumeil \& Chaumeil, 1992)14, et semble répandue au regard des études faites en Amazonie15. Chez les Yanesha, les plantes, indépendamment de leurs spécificités d'usage, qu'elles appartiennent à la catégorie des pare 'shemats, des plantes utiles ou alimentaires, peuvent se répartir soit dans le groupe des plantes cultivées, soit des plantes sylvestres. Pour les Yanesha, il n'existe pas de terme général pour le mot « plante » au sens général d'organisme végétal. La définition de la plante est nettement plus complexe, elle ne peut, en ce sens, se résumer à un seul terme.

Cette étude examine uniquement l'organisation de l'univers végétal des pare'shemats, dans le cadre d'une nomenclature dont les éléments de base ont été fournis principalement par des collectes ethnobotaniques systématiques, doublées de relevés d'herbier et de déterminations botaniques. Dans le cadre de ce travail, 607 échantillons d'herbier ont été collectés, desquels furent identifiés 249 espèces. Notre analyse porte donc sur ces 249 espèces qui comptent à la fois de plantes sylvestres et des plantes cultivées (Bourdy et al., 2008). Cependant, il est certain que ce relevé de données ne put couvrir à lui seul le champ de toutes les espèces reconnues comme étant des pare'shemats, et recenser toutes leurs désignations, soit que les plantes n'aient pu être récoltées, soit que les données livrées aient été parcellaires. En effet, comme il va être expliqué plus loin, les noms des pare'shemats peuvent être constitués par plusieurs termes enchaînés pouvant aller jusqu'à quatre. C'est l'enchaînement complet, qui seul, permet de savoir très précisément de quelle espèce botanique il s'agit. Or, il n'est pas usuel de formuler la dénomination complète pour décrire une plante. Souvent lors des collectes,

${ }^{14}$ Chez les Yagua, il existe des plantes cultivées hátasara et des plantes sauvages towac̃ara.

15 Vincent Hirtzel note aussi cette distinction entre plantes cultivées et sylvestres chez les Yuracaré : «Aymashuñe est donc, sans surprise, le maître des végétaux : il possède des graines de toutes les plantes, aussi bien des plantes "sauvages " qui, en croissant, constituent la forêt, que des plantes cultivées qui deviennent les plantations des humains. » (Hirtzel, 2010). Philippe Descola fait état du couple aramu/ikiama pour expliquer le contraste entre « les plantes cultivées par les hommes et celles qui le sont par les esprits » (Descola, 2005). Pour les Sharanahua, « les plantes [...] qui doivent rester sauvages sont cultivées par les morts», les « plantes cultivées » se nomment fana (Déléage, 2005). Philippe Erikson note que « de nombreux éléments formellement proches des productions de l'activité humaine, mais sans en avoir l'utilité, sont attribués aux maru, en particulier un grand nombre de végétaux sauvages qui évoquent des artefacts ou des plantes cultivées. » (Erikson, 2004). Marie Fleury décrit sur le plan linguistique que « Les plantes portant le suffixe imë sont souvent des végétaux sauvages, le plus souvent inutilisés, contrairement à leurs homologues, parfois domestiqués ou du moins employés par l'homme. Citons l'exemple de napek » (Fleury, 1999). 
et en particulier au début, un seul terme fut donné : soit le plus globalisant, soit le plus signifiant aux yeux de l'informateur, ce qui conduisit quelquefois à une perte de l'information. Le choix du terme qualifiant semble donc variable, nous y reviendrons plus loin. Par ailleurs, cette structure faite d'ensemble long de plusieurs termes permet aussi de comprendre pourquoi plusieurs plantes peuvent être nommées de la même manière lorsqu'un des termes appartenant aux plus englobant les désigne.

La langue Yanesha est polysynthétique de morphologie complexe (Aikhenvald \& Dixon, 2007 ; Daigneault, 2009). Selon Martha Duff-Tripp les composantes de la langue Yanesha sont au nombre de sept : verbe, substantif, adjectif, adverbe, pronom, interjection, et conjonction (Duff-Tripp, 1997). La première racine des substantifs composés peut être un adjectif ou un nom, et peut ainsi s'utiliser indifféremment comme adjectif ou substantif. II existe donc de nombreuses combinaisons de racines adjectives/nominales pouvant former des substantifs/ adjectifs composés. Les mots peuvent donc être des mots simples ou des mots composés. Par exemple, mam désigne « manioc », mampan signifie « un plan de manioc » et nomamar exprime « mon manioc dans la maison ». Dans l'univers des pare 'shemats, chaque espèce botanique se désigne par une succession de termes, dont le nombre peut aller jusqu'à quatre. Chacun de ces termes se compose d'une unité lexicale pouvant être associée (ou non) à un suffixe.

Plusieurs types de suffixes utilisés dans les noms des pare'shemats ont été répertoriés : ceux spécifiant la morphogénèse et ceux se rapportant à un organe végétal. Les suffixes -ech (ou -ach selon le lieu de collecte)16, -nech, -tall, -re $\ddot{c}$, permettent de décrire le végétal dans sa morphogénèse, c'est-à-dire dans son modèle de croissance et de ramification qui lui donnera une silhouette caractéristique. Le suffixe -ech (ou $-a c h$ ) dérive du mot $t$ sach, et s'applique aux végétaux ayant un tronc lignifié, et dont les premières ramifications sont situées dans la partie supérieure, voire à l'extrémité de la personne. Ce suffixe s'applique donc à de nombreux arbres (yonqüellech, Cecropia spp. ; shemaquech, Copaifera paupera (Herzog) Dwyer, également aux palmiers (sech, Euterpe precatoria var longevaginata (Mart.) A. J. Hend.), et aux fougères arborescentes (oshech, Sphaeropteris quindiuensis (H. Karst.) R. M. Tryon). Le suffixe -nech (-nach) caractérise des espèces ayant un axe central non ramifié qui reste vert, et dont les fleurs/fruits se trouvent à cette extrémité : c'est le cas des espèces charrnech, certains Costus spp., ou de I'herbe géante qu'est ch'one'ch (Gynerium sagittatum (Aubl.) P. Beauv.), ou encore tse 'tnach (Vernonia baccharoides Kunth et Vernonanthura patens (Kunth) H. Rob). Le suffixe -tall caractérise les espèces qui, indépendamment de leur taille, ont une tige, lignifiée ou non, qui se ramifie très tôt lors de la croissance de la plante en lui donnant un port arbustif étalé : c'est le cas de atsnoretstall (Begonia spp.), petite plante ripicole, et sottall (Heliocarpus americanus L.), arbre de petite taille. Enfin, -rë̈h désigne invariablement des espèces au port lianescent (aporec̈h, clematis guadeloupae Pers., Llochuore $\ddot{c h}$, 
Smilax poeppigii Kunth). D'autres suffixes sont des marqueurs d'une partie bien précise de la plante, soit en ce qu'elle a de particulier et qui permet de la distinguer d'une autre espèce proche, soit parce que c'est précisément cette partie de plante qui sera toujours utilisée lorsque l'on parle de cette espèce. On dénombre ainsi la résine (-se'), le fruit (-er̃) : Sherencase' par exemple est le latex de l'arbre produisant le caoutchouc, sherencach (Ficus sp.). Le suffixe nominal -eñ provient du mot fruit, chẽ̃. Ainsi corẽ̃ désigne le fruit de la Solanaceae Solanum nemorense Dunal. et aneñtsem̃ celui de Dichorisandra hexandra (Aubl.) Standl. Un des suffixes fréquemment employés est le suffixe nominal -pan, qui se réfère à aspan dont plusieurs sens se complétant mutuellement nous ont été donnés : ce qui est vert, feuille, plante herbacée, petit arbuste, jeune pousse sortant de terre. De fait, ce suffixe s'applique toujours à des espèces de petite taille, telles des petits arbustes du genre Piper et Psychotria, ou des épiphytes sur les troncs des arbres (Columnea spp., Elaphoglossum spp.), ou des herbacées érigées (Costus spp., Anthurium spp.). Entre tous les suffixes, -par est aussi un des plus fréquemment utilisés. Le suffixe -par est dérivé du terme parteñets qui signifie se soigner avec des plantes. Il ne peut être compris que lorsqu'il est accolé à l'unité lexicale : en effet il peut être considéré comme étant un adverbe17 indiquant que l'on parle d'une plante précisément utilisée dans le but d'un soin destiné à l'humain. C'est le cas, par exemple, de cochlle-par (Paullinia bracteosa Radlk.) qui signifie littéralement «qui sert à soigner avec une plante la blessure d'un couteau ». Le suffixe -par s'applique à de très rares exceptions près (errasatspar, Eleutherine bulbosa (Mill) Ur ; pocoyepar, Hedychium coronarium J. König) aux plantes dont les parties aériennes sont utilisées. Le suffixe nominal - par s'applique uniquement aux Cyperaceae (toutes faisant partie du groupe des epe' ou piri piri)18. Cette désignation réfère à p̃arteñets, terme qui signifie « faire en sorte d'avoir, de

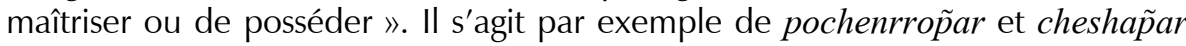
qui, comme tous les souchets, sont utiles pour acquérir certaines compétences spécifiques. Étant donné que l'organe de plante utilisé dans les souchets est toujours le tubercule odorant, duquel sont issues les feuilles caractéristiques, le suffixe - $\tilde{p} a r$ désigne aussi implicitement la partie de plante utilisée. La présence de «suffixes classificatoires » se retrouve aussi au sein de la nomenclature des plantes Ashéninka, groupe également de filiation linguistique Arawak, voisin des Yanesha. De la même manière, ces suffixes insistent « sur des aspects (ou des parties) de la plante » (Lenaerts, 2004).

Par ailleurs, p̃opnor et pasheñorrer, voulant dire respectivement homme/époux et femme/épouse19. Ces deux termes sont des substantifs toujours accolés à un

17 Il s'agit de compléments adverbaux non essentiels ou compléments circonstanciels de but et de manière.

${ }^{18}$ Les termes possédant un suffixe nominal -p̃ar peuvent parfois être succédé du terme queñquehuash tel que le décrit Fernando Santos-Granero (Santos-Granero \& Barclay, 2004), écrit également queñquehuesh par Stéphanie Borios (Borios, 2005).

19 L'existence du couple homme/femme ou mâle/femelle existe aussi chez les Aluku du Haut-Maroni (Fleury, 1999). 
autre terme les précédant et induisent un degré de catégorisation supplémentaire. Ils distinguent deux plantes dont les caractéristiques morphologiques des feuilles sont semblables. Elles se différencient en fonction de leur forme et de leur taille. Par exemple, les feuilles de pa'tcapar p̃opnor (Philodendron sagittifolium Liebm.) sont plus petites et nettement plus sagitifoliées que celle de pa'tcapar pasheñorrer (Philodendron ornatum Schott).

En première lecture, deux grands types de suffixes se distinguent : ceux qui rendent compte d'une caractéristique physique du végétal (modèle de croissance, ou forme de vie, caractéristique physique du végétal), et ceux qui peuvent être considérés comme des adverbes. En s'agglutinant aux unités lexicales, ils indiquent le but recherché (soigner/posséder), et sont des indicateurs indirects de la partie de plante qui sera utilisée lors de l'élaboration du remède (partie aérienne/tubercule). À cela se rajoutent deux substantifs taxonomiques popnor et pasheñorrer, qui complètent et précisent, lorsqu'ils existent, les derniers termes de la dénomination auxquels ils sont toujours rattachés.

\section{1. Agencement des suffixes et définition des modèles de dénomination}

Une des caractéristiques de la dénomination des plantes est d'utiliser un enchaînement ordonné de termes (pouvant aller jusqu'à quatre) pour nommer une espèce végétale, botaniquement identifiée. Un terme peut être construit de deux manières différentes : soit il est constitué selon le modèle unité lexicale — suffixe (U-suffixe) ou bien d'une unité lexicale seule (U)20. Selon les espèces le terme de dernier rang peut être complété par l'adjonction de p̃opnor/pasheñorrer. Il est donc possible de répertorier les différents enchainements des suffixes accolés aux unités lexicales selon leur rang d'apparition dans la déclinaison du nom (rang 1 , rang 2, rang 3, rang 4), et selon la place des suffixes dans la dénomination, différents modèles de dénomination peuvent être mis en évidence (tableau 1).

Une première lecture de ce tableau permet de constater tout d'abord que les suffixes se répartissent dans des rangs différents selon leur nature : les suffixes exprimant les modes de croissance des végétaux (-rec̈h/-ach/-nech/-tall) ne se retrouvent que dans le rang 1 . II en est de même pour le suffixe -pan. Le suffixe -par se trouve, lui, toujours placé en rang deux, trois ou quatre. Le suffixe - par quant à lui est toujours en rang deux ou trois, et est toujours précédé d'un terme sans suffixe. Les suffixes $-s e^{\prime}$ et $-e \tilde{m}$ (résine et fruit) se retrouvent toujours à la fin du nom de la plante et selon le cas peuvent donc être positionnés en rang deux, trois ou quatre. Enfin lorsque dans une combinaison, le premier terme comprend le suffixe -pan, le substantif p̃opnor/pasheñorrer complète le dernier terme. L'observation de l'enchaînement des suffixes indique que c'est le suffixe

${ }^{20}$ Chez les Ashéninka, I'unité lexicale ne se retrouve pas seule, elle est toujours accompagnée d'un « suffixe classificatoire» (Lenaerts, 2004). 
Tableau 1 - Outils de dénomination des pare'shemats

\begin{tabular}{|c|c|c|c|c|c|}
\hline $\begin{array}{l}\text { Modèle de } \\
\text { dénomination }\end{array}$ & Ligne & Terme de rang 1 & $\begin{array}{l}\text { Terme de } \\
\text { rang } 2\end{array}$ & $\begin{array}{l}\text { Terme de } \\
\text { rang } 3\end{array}$ & $\begin{array}{l}\text { Terme de } \\
\text { rang } 4\end{array}$ \\
\hline \multirow{2}{*}{$\begin{array}{l}\text { sans suffixe : } \\
\text { numéro } 1\end{array}$} & 1 & $U$ & & & \\
\hline & 2 & $U$ & $\mathrm{U}$ & & \\
\hline \multirow{3}{*}{$\begin{array}{l}\text { «physique »: } \\
\text { numéro } 2\end{array}$} & 3 & U-(-rec̈h/-ach/-nech/-tall) & & & \\
\hline & 4 & U-(-re $\ddot{c h} /$-ach/-nech/-tall) & $U$ & & \\
\hline & 5 & U-(-rec̈h/-ach/-nech/-tall) & U-(-eñm/-se') & & \\
\hline epe' : numéro & 6 & U & $U$ - $\tilde{p} a r$ & & \\
\hline $\begin{array}{l}\text { pan : numéro } \\
4\end{array}$ & 7 & 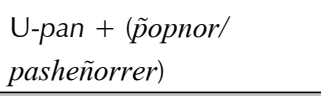 & & & \\
\hline \multirow[t]{4}{*}{$\begin{array}{l}\text { pan-par : } \\
\text { numéro } 5\end{array}$} & 8 & U-pan & 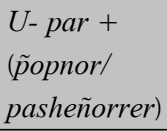 & & \\
\hline & 9 & U-pan & U-par & $\begin{array}{l}\text { U-(-se'/-ẽ̃) } \\
+(\tilde{p} \text { opnor/ } \\
\text { pasheñorrer })\end{array}$ & \\
\hline & 10 & U-pan & U-par & 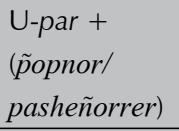 & \\
\hline & 11 & U-pan & U-par & U-par & U-par \\
\hline
\end{tabular}

de rang 1 qui détermine l'enchaînement des suffixes des autres termes. Dans les cas où il n'existe pas de suffixe au rang 1 , il sera vu plus tard que c'est le sens de l'unité lexicale qui commande la nature du suffixe (ou de l'absence de suffixe) à employer au rang 2.

En étudiant les déterminations botaniques, on peut constater qu'il existe en général une très bonne adéquation entre une espèce (au sens d'espèce botaniquement individualisée) et un nom Yanesha. Cette dénomination s'entend dans l'enchaînement intégral de tous les termes. II existe quelques cas particuliers (epe', par exemple) mais qui seront discutés à part. Par ailleurs, il est important de souligner que nos données quelquefois parcellaires sont un obstacle à la généralisation, et leur interprétation peut donc générer certaines inexactitudes.

Une fois ces restrictions faites, en consultant les données botaniques, et en se basant sur les enchaînements de suffixe et le sens des unités lexicales associées, différents modèles de dénomination ont été individualisés : les modèles de dénomination sans suffixe, avec apposition de suffixe " physique », epe', pan, pan-par. 
Dans le modèle de dénomination sans suffixe (lignes 1 et 2), les noms des plantes se déclinent en un seul terme ou en deux termes, I'un complétant l'autre, et agissant comme catégorisant. Par exemple, shemot (rang 1) se décline en shemot Poramasa' poshemoteñ, (shemot du Père des vautours, Lycopersicon peruvianum var. peruvianum), shemot Huare'shemot (shemot de la Divinité Huar, Physalis pubescens L.), sha'rẽ̃ po'shemoteñ (shemot du lézard, Physalis sp.), tsem po'shemoteñ (shemot d'une espèce de vautour, Solanum stramoniifolium Jacq). Ce groupe comprend dans sa majorité des espèces cultivées ou domestiquées 21 à des fins alimentaires ou rituelles, même si certaines ont été parfois citées comme utilisées à des fins médicinales (ex. ̈̈ho, Dioscorea triphylla Buch. Ham. ex Wall. ; c̈hop, le maïs ; mats, Tephrosia sp. ; yetsep, le roucou, Bixa orellana L.). Il faut noter que certains des noms relevés dans cette catégorie sont dérivés du Quechua : patohuare' (Tabernaemontana sananho Ruiz \& Pav.), shemochuarep (Tessaria integrifolia Ruiz \& Pav..) tohuan (Bromelia sp.), coc', la coca, ou encore chemuer (Mussatia hyacintina (Standl.) Sandwith.), ce qui signale peut-être un emprunt ou du moins un usage qui s'étend au-delà des frontières Yanesha. Par ailleurs, les plantes dont la dénomination ne possède pas de suffixe sont généralement des états transformés de Divinités ou d'ancêtres.

Dans le modèle de dénomination avec suffixe «physique », le terme de rang 1 porte le suffixe $(-r e \ddot{c h} /$-ach/-nech/-tall). À ce niveau, soit le nom Yanesha ne comprend qu'un terme de rang 1 et correspond à une espèce botanique dûment individualisée et identifiée (ligne 3 ), soit le rang 1 inclut plusieurs espèces botaniques. Dans le premier cas, les plantes sont, comme précédemment, souvent issues de Divinités ou d'ancêtre. Dans le cas où le rang 1 inclut plusieurs espèces botaniques (ligne 4 et 5), la distinction entre les espèces est rendue opérative par l'usage du terme de rang 2 qui va à son tour préciser une caractéristique physique du végétal en ce qu'il a de particulier et de différent qui peut être indiqué par I'utilisation d'un suffixe (-ese', -eñ), ou bien qui va être un autre substantif sans suffixe. Ces substantifs sans suffixes sont des noms d'animaux, ou bien précisent un usage. Par exemple topomech (rang 1) correspond à trois espèces de Guarea (G. guenteri, G. macrophylla, G. sp.) respectivement identifiées par les termes de rang 2 comme topomech yanosoten (espèce de luciole qui ne vole pas, Guarea guentheri Harms), topome'ch matsemech (de la nivrée nommée barbasco, Guarea macrophylla Vahl) et topome 'ch ashateclle' (colombe à œil rouge, Guarea sp.). II en est de même pour Vismia baccifera (L.) Triana \& Planch, Vismia confertiflora Spruce ex Reichardt, et Vismia sp. dont le terme de rang 1 est pour toutes les espèces sorroyarečh, et qui se distinguent ensuite par les substantifs employés dans les termes de rang 2 ; la même remarque peut être faite pour les espèces de Urera (U. baccifera et Urera spp.) toutes désignées par le terme de premier rang sho'reč h, et individualisées comme espèces par un terme de deuxième rang sans suffixe. Le sens des unités lexicales associées aux suffixes -rë̈h/-ach/-nech/-tall est aussi toujours un descripteur du monde tangible. Ces unités lexicales associées se réfèrent parfois à un animal comme topom-ech (la colombe), sot̃-ech (poisson

21 Dans le cas de plantes cultivées, il est certain qu'il existe d'autres termes complémentaires désignant les variétés, mais que nous n'avons pas relevés. 
de la famille des Loricariidae, carmarcha en espagnol régional), shemocua-rec̈h (espèce de martin pêcheur), ou à une caractéristique physique du végétal luimême telle Yonqüell-ech (cavité creuse), ou bien à un critère organoleptique comme pesher-rec̈h (amer), charn-ech (acide), ou encore à des symptômes tels ollo'charets-rë̈h (vers intestinaux), sho'r-ech (shorañets : avoir des boutons), astsornets-all : aigreur (d'estomac), ou bien au nom de personnages mythiques Huallapnarr-ech (Divinité de rang inférieur, Huallapnarrena') tse' $\square n$-ach (ancêtre de rang hiérarchique supérieur, servant de Yompor Huar). D'un point de vue botanique, les espèces appartenant à ce groupe constituent un fond hétérogène, d'arbres, d'arbustes, de lianes, d'herbacées, poussant en bord de rivières ou formant en partie la végétation secondarisée, assez faciles à trouver et relativement abondantes. Très peu d'espèces de forêt primaire semblent en faire partie. D'un point de vue ontologique, les plantes dont les unités lexicales associées aux suffixes -re $\ddot{c h} /-$ ach/-nech/- tall sont souvent des plantes dont l'origine est une sécrétion de Divinité de rang hiérarchique intermédiaire ou une sécrétion d'ancêtre de rang supérieur, et dans ce cas-là, le suffixe signale la partie de plante à utiliser pour préparer le remède dont la nature est identique à la sécrétion d'origine, ou bien on a affaire à des plantes issues d'état transformé d'ancêtres, ou des Divinités de rang généalogique inférieur. Ce modèle de dénomination pourrait être décrit comme un « modèle physique », car c'est un ensemble de caractéristiques du domaine physique, perçu et lié au végétal, qui semble primer et être utilisé comme outil taxonomique, mais cette appréciation physique est sous-tendue par une raison, celle du mode d'apparition du végétal dans le monde « visible ».

Un autre modèle de dénomination peut être individualisé, ayant pour base I'utilisation dans le premier terme du suffixe -pan mais ne possédant pas de second terme avec le suffixe -par à la différence des lignes suivantes. Dans ce modèle, le nom de la plante ne comprend qu'un seul terme (avec éventuellement la présence du substantif p̃opnor/pasheñorrer), et les unités lexicales associées à -pan font allusion à une caractéristique de la plante, une symptomatique précise ou désignent un personnage mythique, par exemple : arota-pan (tourterelle), ollamepan (œuf de pou, en rapport avec le bruit de la graine de la plante écrasée), morocsheñtso-pan (avoir une éruption cutanée), omuerets-pan (petit-fils de Yato' Mopoll, état transformé de Divinité de rang inférieur), añechech-pan (servante de Yachor Santosapuecoya, état transformé d'ancêtre de rang hiérarchique inférieur). Ce modèle de dénomination peut se rapprocher du modèle de dénomination « physique » décrit précédemment. Commençant aussi par le suffixe -pan employé dans le premier terme, un autre modèle de dénomination apparaît. Le suffixe de rang 1 est toujours -pan, et le deuxième terme est toujours le suffixe -par (exprimant l'usage que l'on fait de quelque chose). Une variabilité apparaît dans le terme de rang 3, qui peut comprendre à nouveau le suffixe -par, ou bien être une unité lexicale simple, ou encore avec un suffixe exprimant une caractéristique physique de la plante $(-s e \%-e \tilde{m})$. Le dernier terme finalisant la dénomination de la plante peut être pasheñorrer et p̃opnor (« de l'homme/époux » / « de la femme/épouse ») et induit un nouveau degré de catégorisation. On trouve par exemple : corarnopan challacochnapar o'muerestspar pasheñorrer (Piper sp.) 
et corarnopan challacochnapar o'muerestspar popnor (Piper umbellatum L.). Dans ces groupes, il existe une autre particularité de dénomination. En effet, il y a toujours une espèce dont les deux derniers termes sont identiques (ex. campuerpan choyeshe'matspar tsapo'marneshapar tsapo'marneshapar, Piper sp. ; campuerpan choyeshe'matspar choyeshe'matspar, Piper augustum Rudge). Dans ce cas, c'est le sens des unités lexicales associées aux suffixes -pan et -par qui permettent de comprendre la logique du système de catégorisation. Les unités lexicales associées à -pan ne sont pas très nombreuses : elles désignent soit un mode thérapeutique campro-pan/campuerr-pan (bain de vapeur), soit l'acte de soigner en lui même utilisant l'unité lexicale corar- qui pourrait être un dérivé de curar (espagnol : soigner, selon certains informateurs « corar » désignerait une petite grenouille), soit sont explicitement les noms de certaines entités étiologiques issues de Yosoper (ex. errasañatspan). Les unités lexicales associées à -par peuvent se référer de manière littérale à une voie d'administration, un symptôme ou un syndrome, un mode d'affectation de la personne (exemple gac̃hatentso-par : s'effrayer de voir quelqu'un qui disparaît), ou se réfèrent de manière métaphorique à une «maladie ». Dans ce cas, la cause de la «maladie » se confond avec le nom de la «maladie » (exemple : ellapa-par littéralement « qui sert à soigner les hémorragies provoquées par un fusil (ellap) ayant été « soigné » pour retrouver sa fonctionnalité de chasse »), ou la cause de la « maladie » reprend directement le nom d'une entité étiologique et/ou les conséquences symptomatiques du mal que celle-ci provoque (ex. erratsañats-par, Erratsañats : entité du monde «non visible » et envoyé de Yosoper, veut dire à la fois hémorragie, et représente l'entité étiologique qui provoque des hémorragies).

De fait, en combinant le modèle de dénomination et le sens des unités lexicales, il apparait que les plantes de modèle de dénomination -pan/-par partagent toutes la même origine ontologique. Il s'agit de plantes données par des entités du monde « non visible » tels les mellañoteñ, les atopenets et les entités « de passage », qui à leur tour serviront à soigner les maux qu'elles occasionnent. Le suffixe -pan s'accolant à une unité lexicale est significatif de plantes issues de dons d'entités : campuer-pan (campror-pan), outre le fait d'avoir la signification de bain de vapeur, signale aussi toutes les espèces données par les mellañõ̃eñ, qui traiteront les entités étiologiques envoyées par les mellañoteñ même et celles du monde « non visible», celui « de la nuit », et corarno-pan, regroupe toutes les espèces données par les mellañoteñ et qui soignent les entités étiologiques du monde « non visible » et «qui marchent de jour ». De la même manière errasañatspan représente le groupe de plantes données par Errasañats pour soigner les hémorragies, etc. Dans ce modèle de dénomination, les termes auxquels est accolé un suffixe exprimant une caractéristique physique de la plante servent à faire une différence permettant de distinguer deux espèces proches, auxquelles I'adjonction de p̃opnor/pasheñorrer induit un niveau taxonomique supplémentaire, mais non obligatoire 22.

22 D'autres plantes forment également de petits groupes, mais ils sont en nombre nettement moins important et les précisions à leur égard sont relativement vagues, ces groupes tels añechechpan, 
Il est à noter que d'un point de vue botanique les espèces appartenant au groupe campuer-pan (campror-pan) sont toutes des espèces soit du genre Piper, Psychotria, Costus, ou encore Elaphoglossum, et que les corarnopan sont dans leur plus grande majorité des espèces de Piper (90\% des plantes déterminées). Une notable homogénéité botanique se dégage donc de ces deux groupes de plantes, qui partagent aussi la caractéristique - du moins pour les Piper spp. — d'être souvent aussi très semblables entre elles d'un point de vue morphologique. Elles sont également toutes aromatiques corarnopan. Cette observation est peut être à rapprocher de celle faite pour les epe' : des similitudes morphologiques aussi poussées signent les volontés d'une Divinité suprême ou des êtres qui lui sont directement liés. Cependant cette observation d'uniformité botanique s'appliquant au niveau du genre n'est pas valide pour les autres groupes des plantes données par d'autres entités étiologiques.

Ainsi, il est possible de distinguer des modèles de dénomination exposés, trois systèmes nomenclaturaux distincts qui sont directement corrélés à l'origine ontologique des plantes. Dans le premier, les plantes issues de transformation de Divinités/ancêtres possèdent une dénomination dont l'enchaînement des termes est restreint à un voire deux présentant un suffixe ou non. Issues de transformation, ces plantes restent organisées selon le même schéma de parenté de la Divinité ou de l'ancêtre duquel elles proviennent. Dans ce système de parenté, une plante n'est donc pas strictement liée à d'autres plantes. C'est le cas, par exemple, de Yo'c̈h Huallapnarrenana' devenue huallpnarrec̈h (Munnozia hastifolia (Poepp.) H. Rob. \& Brettell) et dont sa Mère, Yachor Santosaprecoya, est aujourd'hui présente sous forme d'une étoile. Le second système nomenclatural est celui des epe', plantes données par Yato' Yos ou par certains Cornesha'. Enfin, à ces deux modèles, s'ajoute celui des plantes données par des entités du monde «nonvisible ». Ces plantes possèdent une dénomination complexe qui fait intervenir plusieurs termes et dont nous allons considérer la signification.

\section{SIGNIFICATION DES NOMS DE PLANTE}

L'attention est ici portée sur les plantes dons de mellañoteñ nommées campuerpan et corarnopan. En ce qui concerne, les campuerpan l'enchaînement des termes et la signification des unités lexicales s'organisent comme le mentionne la figure 2. L'unité lexicale de rang 1 commune à tous campuerpan est campuer- (inhalation de vapeur émanant de plantes chaudes bouillies) et détermine le mode d'administration du remède. Les unités lexicales de rang 2 telles atatasñats- (rencontre avec une entité étiologique), choyeshe'mats- (ombre errante), gac̈ha'teñets- (voir une personne qui disparaît instantanément) ou encore Muec̈ho' $\tilde{t}$ - (Père des singes envoyé des mellañoteñ) réfère communément au mode d'affectation de la personne ou à une

chellca'pan ou encore puepa'tpan, semblent assez méconnus et n'ont pas été retenus dans notre analyse. 


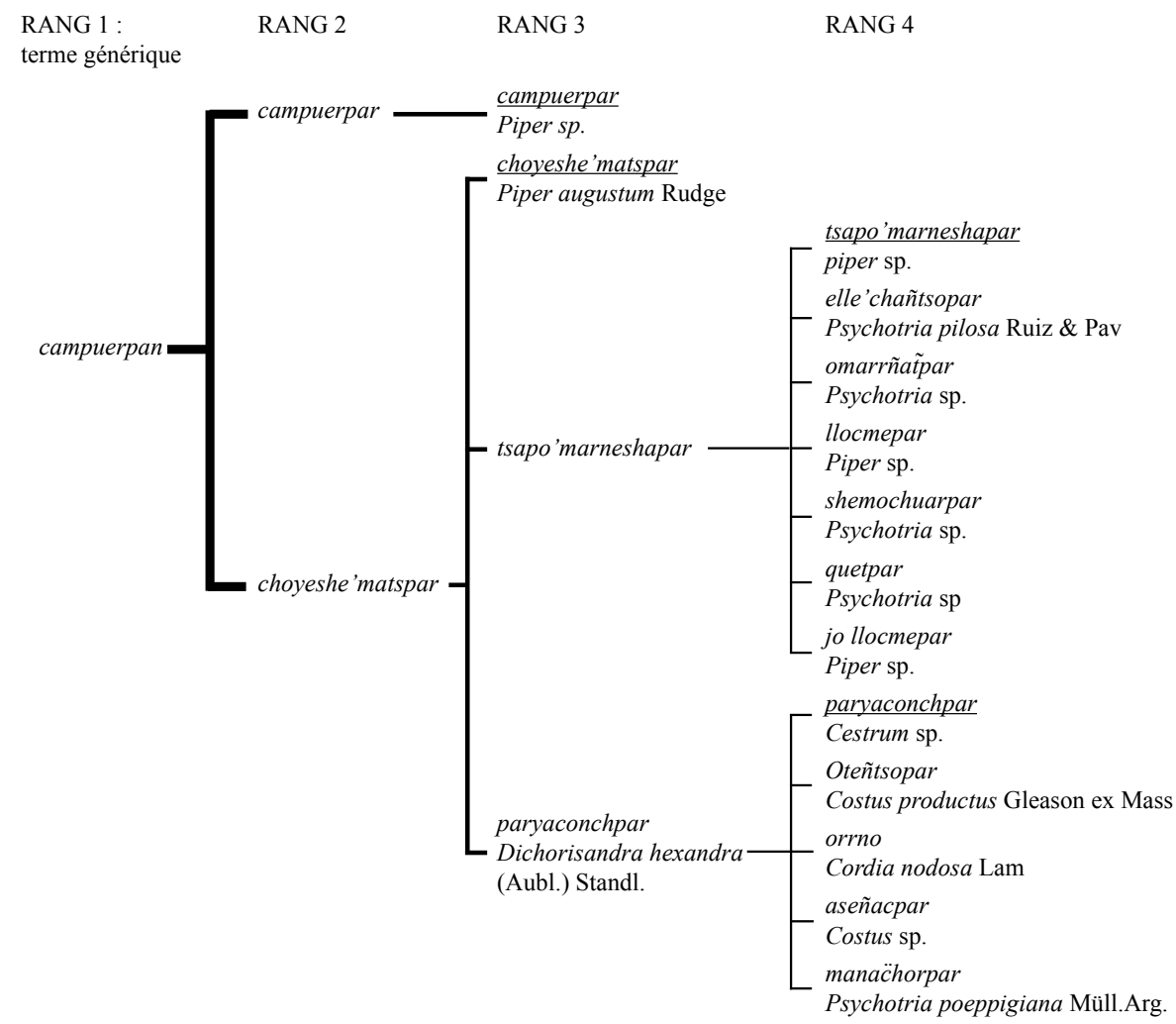

Plante dite «légitime»

Figura 2 - Système nomenclatural de campuerpan

entité étiologique précise de pathogénie connue. Par ailleurs, les unités lexicales de rang 3 mentionnent des effets thérapeutiques recherchés, des causalités précises d'un mal ou encore des manifestations spécifiques à certaines entités étiologiques. Ainsi, che 'llec- signifie " être uni », Paryaconch- est une entité étiologique diurne provoquant des mouvements d'air et Tsapo'marnesha'- désigne l'ensemble des entités étiologiques qui marchent la nuit. Enfin, le dernier terme exprime soit le nom d'une entité étiologique soit une de leurs manifestations connues lorsqu'elles prennent forme dans le monde «visible ». Dans le premier champ sémantique de ce rang 4, se trouve par exemple Jo-, entité étiologique marchant de nuit et Elle'chañ, le Père des daguets rouges. La signification de l'unité lexicale désigne donc directement la causalité de la «maladie » pour laquelle est utilisée la plante. Le deuxième champ sémantique rencontré fonctionne par analogie, par exemple llocmets- (articulation) manifeste ici l'os de fémur toujours transporté par Jo', aseñac- (Colibri de bec tordu) signifie « colibri devançant un chaman ayant une 
tendance à faire de la sorcellerie », ou encore manac̈hor- (murmure du vent dans les arbres) et orrno- (souffle de vent brutal) sont les marqueurs de présence de certaines entités étiologiques se déplaçant la journée.

Dans la catégorie corarnopan, les unités lexicales des termes de rang 2 font référence aux cornesha' mythiques Cachenarena' et Challacochnachena' ayant reçu des plantes afin de soigner les Yanesha (fig. 3). Les significations des unités lexicales des termes de rang 3 renvoient souvent à des entités étiologiques diurnes en lien avec les climats ou les lieux humides. Dans cette catégorie de plantes, les suffixes $\tilde{p}$ opnor/pasheñorrer semblent presque systématiquement toujours employés et terminent chaque dénomination. Pour leur part, les corarnopan ont pour une unité lexicale de rang 1 corar- désignant, au même titre que l'unité lexicale de rang 1 des campuerpan, le mode d'administration. Les unités lexicales de rang 2 désignent soit le destinataire ontologique du don, comme cachenarena'-, cornesha' qui reçut le don de soigner, soit le mode d'administration de la plante comme señts- sortir quelque chose de chaud (jus chaud des feuilles bouillies). Au dernier rang, l'unité lexicale exprime la causalité d'un mal (Ayon-, arc-enciel), l'effet thérapeutique recherché (huomenc-, être vaillant, fort), le mode d'affectation de la personne (mecha'teñets-, avoir peur) ou nomme directement une entité étiologique diurne (Omarrñat-). La signification des unités lexicales varie donc selon la nature de l'entité ayant fait le don et partant de là, selon la nature de l'entité étiologique qui sera traitée avec la plante. Si dans les cas des entités nocturnes (donc invisibles) il est utile de préciser les signes révélateurs de leur présence (murmure du vent dans les arbres, mouvement d'air violent, oiseau de mauvaise augure), cela ne sera plus nécessaire dès lors que l'on parlera d'entités qui marchent de jour que l'on caractérise directement par leur nom, étant donné qu'elles sont généralement trouvées lors de phénomène atmosphérique ou dans des lieux bien particuliers. Les unités lexicales utilisées dans ces deux cas peuvent être compris comme des termes mnémotechniques, aidant à faire le diagnostic de l'entité impliquée, l'effet thérapeutique recherché, et le mode d'administration du remède. L'ordre dans lequel se trouvent les unités lexicales semble variable.

Au sein de la catégorisation, une plante dont l'unité lexicale est identique à chaque rang de sa dénomination est dite « légitime »23. Elle est dotée d'un pouvoir thérapeutique supérieur à toutes les autres de même rang et peut donc soigner toutes les affections susceptibles d'être provoquées par des entités étiologiques. Dès lors, il est possible de définir à l'intérieur de chaque sous-catégorie des plantes dont l'usage est spécifique à un mal précis, et une plante " légitime », sorte de joker connu pour soigner toutes les affections pouvant être soignées par les plantes de cette sous-catégorie.

En résumé, les agencements des unités lexicales correspondant aux plantes dons de mellañoteñ campuerpan et corarnopan, sont sensiblement identiques. Les unités lexicales de rang 1 référent toujours au mode d'administration, alors que au

23 Le terme « légitime » est la traduction littérale de legítimo employé par les Yanesha pour désigner ces plantes de pouvoir thérapeutique supérieur. 


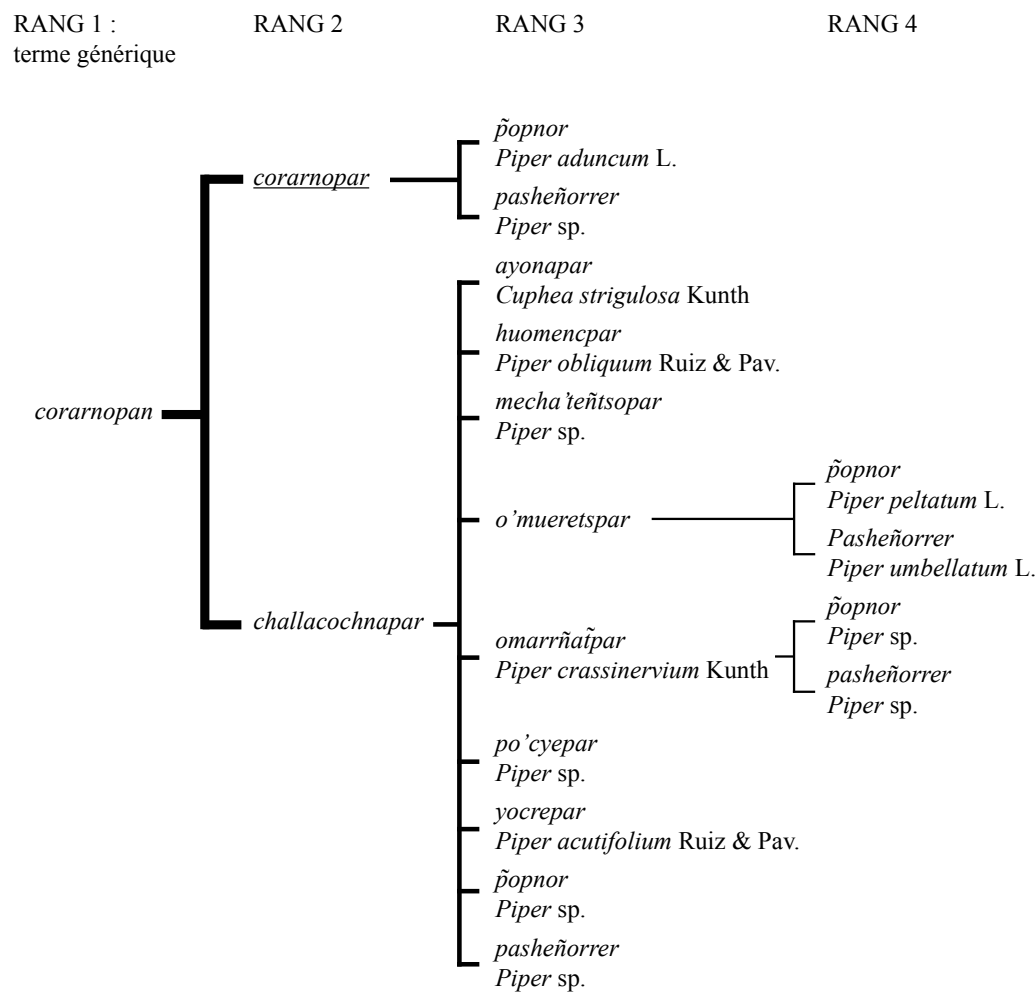

Plante dite «légitime»

Figura 3 - Système nomenclatural de corarnopan

rang 2 et 3 les significations divergent. Au rang 2, elles renvoient respectivement aux modes d'affectation de la personne et au mode d'administration tandis qu'au rang 3, les unités lexicales de campuerpan et corarnopan expriment les effets thérapeutiques et/ou le nom d'une entité étiologique. Une autre variante d'agencement des unités lexicales de ces deux groupes de plantes est l'absence de terme au rang 4 de la dénomination des corarnopan, alors que les plantes que regroupe le terme campuerpan ont une unité lexicale de rang 4 exprimant de manière analogique l'une des manifestations d'une entité étiologique précise lorsqu'elle prend forme dans le monde «visible». Considérant alors que ces plantes sont originaires de don divin, des désignations spécifiques font sens et laissent apparaître un système nomenclatural bien étroitement lié aux origines ontologiques des plantes.

D'un point de vue comparatif, il est possible de constater des similitudes avec la catégorisation des plantes Ashéninka. En effet, la signification des unités lexicales est principalement « déterminée par l'usage attribué à la plante » (Lenaerts, 2004). De même, les significations des unités lexicales de rang 1 et 2 font peu référence aux caractéristiques morphologiques des végétaux. Certaines unités 
lexicales d'autres catégories de pare'shemats (errasañatspan et yonnañetsopan), expriment, comme chez les Ashéninka, des sécrétions corporelles comme le sang ou encore des saveurs comme l'amertume. Par contre, certaines significations des unités lexicales de rang 3 et 4 expriment un système analogique définissant des correspondances entre le nom de la plante, la morphologie végétale et l'usage. Les informations fournies au pied de la plante, lors des récoltes, expliquent souvent des caractéristiques morphologiques affirmant ainsi qu'il s'agit bien de la plante recherchée. Par exemple, jo llocmepar est un Piper dont la tige présente des nœuds disposés à intervalle régulier. Un tronçon de tige ressemble à un os dont les articulations seraient formées par les nœuds, zone de renflement de la tige. De même, ayonapar (Cuphea strigulosa Kunth) possède des fleurs dont les teintes varient du pourpre ou rose et une tige dont la couleur est également changeante, passant du vert foncé au rouge bordeaux au niveau des plus petites ramifications. Enfin, huomencpar (Piper obliquum Ruiz \& Pav.) possède des feuilles relativement coriaces/solides tout comme l'effet thérapeutique recherché : huomenc- signifiant fort et vaillant. Ces caractéristiques analogiques se retrouvent dans certains groupes linguistiques Pano. Chez les Yora par exemple, les fleurs de Peperomia sont préconisées pour traiter les épistaxis. Ces fleurs ressemblent à la queue des singes Saïmiri dont « l'esprit » est connu pour provoquer des saignements de nez (Shepard, 1999).

\section{PRINCIPE DE COHÉRENCE ET LOGIQUE DIAGNOSTIQUE}

Au sein de la société Yanesha, tout le monde connaît des plantes médicinales. Cependant, il existe des acteurs de soin spécialisés : pa'llerr, le chaman « preneur de tabac » et apartañ, le(la) végétaliste (Smith, 1977 ; Santos-Granero \& Barclay Rey de Castro, 2004). Les plantes utilisées en réponses thérapeutiques à une étiologie proposée comme causalité a priori, telles que les atsnañets, sont des pare'shemats. Elles sont souvent préconisées par des membres de la famille ou le cas échéant par le végétaliste, lorsque la situation est jugée grave. Par ailleurs, l'acteur de soin privilégié en cas d'aggravation majeure de l'état de santé ou d'affections liées à un acte de sorcellerie sera le pa'llerr. Lorsqu'il intervient, le tabac représente la principale plante utilisée. Dans ce cas, ainsi que le mentionne Marc Lenaerts « le chaman n'a pas nécessairement besoin d'être un bon botaniste » (Lenaerts, 2006). Ainsi, les affections provoquées par les entités étiologiques sont principalement prises en charge par les végétalistes : elles se soignent principalement à l'aide de plantes.

En ce qui concerne le raisonnement diagnostique, les entités étiologiques sont suspectées lorsque la personne manifeste un état dégradé de santé sans douleur corporelle localisée. Cette quête de sens passe par une anamnèse permettant d'identifier l'être surnaturel à l'origine des symptômes ressentis ou exprimés. Cela permet ensuite de faire un choix thérapeutique adapté. Par la suite, c'est la nature de l'entité étiologique révélée par le biais du diagnostic qui définit le choix de la plante. En effet, comme cela a été dit, nommer l'entité, c'est déjà nommer une plante. À leur tour, les plantes sont réunies dans de grands groupes et chacune 
des espèces les composant est issue de dons de mellañoteñ ou d'añóteñets. À chaque groupe de plantes donné dans un but précis, correspond donc une catégorie d'usage : les groupes de plantes désignées par le terme de campuerpan et corarnopan sont spécifiquement dédiés aux conséquences des rencontres avec une entité étiologique (fig. 4).

Le principe vital arraché à la personne à la suite d'une rencontre avec une entité étiologique (appartenant au groupe des tsapo'marnesha', des ashcatañ, les envoyés des mellañoteñ, ou encore certaines entités étiologiques diurnes, yet̃o'marnesha) ou décollé du corps par perte d'adhésivité après une frayeur subite (que provoquent une ombre errante ou certaines entités étiologiques diurnes), positionne la personne dans la nécessité d'un soin. Campuerpan est la catégorie de plante systématiquement employée dans ce cadre. L'étymologie du nom de ce groupe de plante se réfère à campuerr, procédé thérapeutique qui consiste à inhaler de la vapeur et prescrit très exactement la manière dont elles doivent toutes êtres administrées. Les plantes qui vont donc être utilisées dans les affections induites par des tsapo 'marnesha' ou des ashcatañ, font toutes partie du groupe de plantes de terme générique campuerpan. Si l'entité étiologique nocturne est connue d'emblée ou suspectée, le choix de la thérapeutique se porte vers le végétal qui lui correspond. En effet, il existe une correspondance étroite entre l'entité étiologique nocturne et la plante utilisée. Par exemple, à une personne qui aurait été affectée par Jo', il est nécessaire de lui administrer jo'llocmepar (Piper sp.). De même, lors d'une atteinte portée par Omarñets, le choix thérapeutique se tourne vers omañatspar (Psychotria sp.). Si au contraire, il est impossible de déterminer avec précision l'entité étiologique, il est préférable d'utiliser la plante considérée comme « légitime» au sein de son groupe, celle dont le terme de rang 4 est tsapo 'marneshapar (Piper sp.). Cette espèce, qui porte ellemême le nom générique globalisant de tout un groupe d'entités est considérée comme extrêmement puissante, d'un point de vue thérapeutique, capable de soigner " n'importe quelle maladie, toutes celles qui sont dues aux démons », soit n'importe quel tsapo'marnesha'. C'est aussi à cette plante que l'on pensera lorsque l'état de la personne s'aggrave, soit suite à la survenue de symptômes secondaires qui l'affaiblissent fortement, soit par aggravation de symptômes « d'appel », ce qui porte le doute sur l'identité exacte de l'entité ayant été cause de la «maladie » et demande une reconsidération partielle de diagnostic. Enfin, dans les cas extrêmement préoccupants, il devient nécessaire de recourir à des plantes telles que choyeshe'matspar (Piper augustum Rudge), pour empêcher le détachement du yechoyeshe 'm, et, par extension, permettre d'éviter l'éloignement du principe vital. Enfin, quand la frayeur subite est imputée à des yetõ'marnesha' dites « du vent » telles Paryaconch ou Aptallesah' suite à un mouvement d'air soudain provoquant la chute d'un arbre, par exemple, les plantes préconisées sont également des campuerpan telles paryaconchpar (Cestrum sp.). Ainsi, les plantes utilisées pour pallier à un état généralisé de souffrance provoqué par une entité étiologique, qu'elle soit tsapo'marnesha', ashcatañ, choyeshe'mats ou encore entité étiologique diurne, ñorranesha', sont spécifiques à leurs étiologies respectives, et appartiennent toutes à la catégorie des campuerpan. Dès lors que 
Céline Valadeau

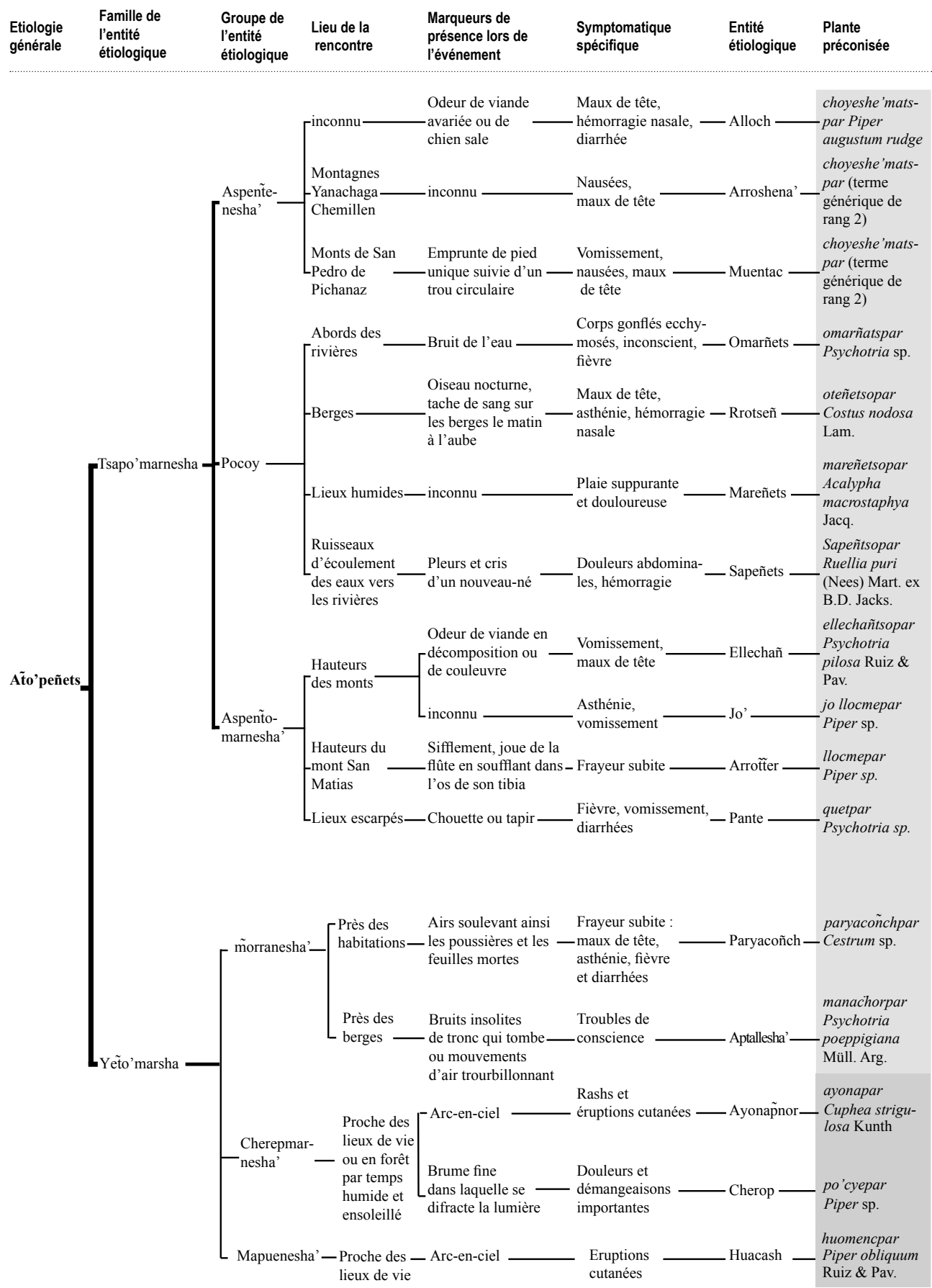

campuerpan

corarnopan 
le raisonnement diagnostique permet de découvrir l'agent étiologique, le remède et sa formulation se connaissent spontanément. Cependant, même s'il existe un lien étroit entre espèce et entité, donc une spécificité d'usage pour chaque plante, une certaine marge est laissée lorsque l'entité n'est pas connue ou que l'on doute du diagnostic. II est alors fait appel à une plante dite "légitime » qui possède la capacité de soigner indistinctement n'importe quelle atteinte de n'importe quelle entité du groupe auquel elle appartient. La restitution de principe vital à la personne ne dépend alors pas de la quantité de végétal utilisée dans le remède, mais de la puissance intrinsèque de la plante, en d'autres termes de sa position au sein de l'arbre nomenclatural. Dans ce cas, la puissance maximum de la plante est attribuée à celle ayant le spectre d'action thérapeutique le plus étendu. II n'est donc pas toujours nécessaire de connaître avec exactitude l'étiologie pour recommander une plante.

Tangibles par leurs manifestations visibles sur la peau de la victime, les affections cutanées sont souvent la preuve qu'une rencontre avec une entité étiologique diurne - dont les plus connues sont celles de l'arc-en-ciel et de l'eau - a bien eu lieu. Quelle que soit l'entité, toutes ces affections se soignent avec des plantes appartenant au groupe des corarnopan. Les affections de l'arc-en-ciel se soignent donc systématiquement avec des corarnopan, et plus précisément des plantes dont le terme de rang 2 est challacochnapar. Parmi celles-ci, ayonapar (Cuphea strigulosa Kunth) et po'cyepar (Piper sp.) sont utilisées dans les soins respectifs des affections dues à Ayona ̃̃nor et Cherep. Les troubles cutanés provoqués par Huacash peuvent être traités avec la plante huomnecpar (Piper obliquum Ruiz \& Pav.). Toutes ces plantes sont utilisées dans le but d'extraire les principes vitaux injectés dans la peau par les entités. C'est pourquoi elles sont toutes utilisées en cataplasme. II en est de même pour les furoncles, souvent engendrés par Omarñets et traités avec des cataplasmes chauds de plantes appartenant au même sous-groupe challacochnapar, appelées omarrñatp̃ar p̃opnor et pasheñorrer (Piper crassinervium Kunth ou Piper spp.) ou encore po'cyepar (Piper sp.). II est dit que « le liquide qui est dans la tumeur (le furoncle) grandit, il faut le faire sortir. Pour cela, il faut mettre des plantes dessus, car elles savent sucer le mal afin que la tumeur éclate ». Dans ces étiologies, il est également possible d'avoir recours à des plantes dites « légitimes», lorsque se formule un doute sur l'origine du mal, ou que la situation s'aggrave. Elles sont au nombre de deux (corarnopar p̃opnor et corarnopar pasheñorrer : Piper aduncum L. et Piper sp.) et décrites comme « épouse/ femelle et époux/mâle ». Elles doivent être utilisées ensemble pour être efficaces.

Ainsi, l'identification de l'entité étiologique mise en cause dans l'état dégradé de la personne est un processus progressif. En suivant les deux exemples précédents, il est possible de constater qu'à chaque étape de l'identification de l'étiologie (par exemple : atsnañets, tsapo 'marnesha', aspento'marnesha', Pante) la dénomination de la plante change (campueran, choyeshe'matspar, tsapo'marneshapar, quetpar). Au fur et à mesure de l'anamnèse, les terminologies employées progressent des dénominations les plus globalisantes vers les plus précises. Cela explique pourquoi la nomenclature végétale ne s'explicite jamais dans son intégralité - quatre termes successifs - lorsqu'il s'agit de nommer une plante. La dénomination de 
la plante est ainsi intimement liée à son usage à un moment précis. Dès lors, la dénomination employée pour définir une plante possède de l'intérêt parce qu'elle s'intègre dans un processus dynamique. Ainsi, au lieu de considérer qu'une plante se dénomme grâce à un enchaînement de quatre termes successifs, il semblerait plus exact de dire qu'une même plante possède plusieurs noms. Le nom de la plante est donc choisi au moment de l'évènement, la conjoncture précisant sa dénomination. Ainsi, les supra-catégories ne seraient pas ici, comme chez les Ashéninka, « de véritables catégories englobantes, mais plutôt des sortes de butoirs à la pensée, qui permettent d'oublier momentanément des ramifications sans intérêt actuel » (Lenaerts, 2006).

\section{CONCLUSION}

Chacun des termes associés aux plantes est constitué d'une unité lexicale à laquelle un suffixe peut être ou non ajouté. Ces suffixes sont caractéristiques du monde végétal et ne s'appliquent qu'aux plantes. Deux grands types de suffixes ont été recensés : ceux qui décrivent une caractéristique physique liée à la morphogénèse du végétal, et ceux qui peuvent être considérés comme des adverbes précisant le sens de l'unité lexicale qui les accompagne. Les suffixes décrivant les caractéristiques «physiques » peuvent être quelquefois considérés comme ayant une fonction taxonomique, mais dans la plupart des cas, l'ensemble unité lexicale/suffixe remplit cette fonction. La manière de combiner une succession de termes composés d'un suffixe et d'une unité lexicale signifiante permet de définir des patrons de dénomination des plantes. Ces modèles de dénominations individualisent de grands groupes de plantes ayant une origine ontologique commune : les plantes issues des dons d'entités étiologiques, les plantes issues des transformations d'ancêtres, et celles émanant de la volonté directe de Yato' Yos et de certains cornesha'. La catégorisation Yanesha des pare 'shemats en son sens général apparaît donc être basée sur des critères ontologiques.

La manière de nommer les plantes, quant à elle, reprend directement le processus d'apparition de la plante dans le monde « visible ». Ce processus peut être lié à une énonciation verbale (des plantes données par des entités), une transformation physique (des plantes états transformés d'ancêtres), ou bien une émanation directe de la volonté de Yato' Yos (catégorie epe'). En d'autres termes, nommer une plante c'est répéter le processus ayant donné lieu à son apparition. C'est le mode de formulation du remède qui recréé ensuite la forme originelle de la plante. Elle s'administre alors telle une composante de la personne dont elle est issue. Ainsi, la plante n'est qu'une forme déguisée d'une forme ontologique (corps/sécrétion/ intention-souffle vital), un état de transformation transitoire. Cette forme restituée lors de la formulation vise à humaniser l'être, lui permettre de continuer à user du souffle vital insufflé au stade fœetal. Si l'humain cherche à rester humain, c'est dans la plante qu'il trouve une base d'humanité. La plante doit donc être considérée dans une sorte de continuum qui passe de l'ancêtre/Divinité en somme humain des temps primordiaux, à la forme végétale, aux remèdes c'est-à-dire à la forme 
ontologique dont elle est issue, à la personne. Il ne distingue finalement pas I'humain de la plante puisqu'elle même en est une de ses composante. Par ailleurs, il existe des liens de cohérence très précis entre l'évènement de la «maladie», l'étiologie, l'état dégradé de santé de la personne (soustraction ou addition de yecamquëm), la réponse thérapeutique de la plante (additive ou soustractive en terme de principe vital) et la formulation et la dispensation du remède. Le système de catégorisation des plantes, celle des entités étiologiques ainsi que l'organisation sociale des Yanesha sont semblables, elles organisent un système hautement cohérent qu'explicitent les structures et les agencements linguistiques.

En considérant d'autres travaux portant sur les catégorisations vernaculaires d'autres groupes amazoniens, il est possible de constater certaines dissemblances. Ces dissemblances se situent essentiellement au niveau du mode de catégorisation. En effet, tout comme la taxonomie des plantes Ka'apor (Balée, 1989), les plantes Shipibo-Konibo s'organisent volontiers selon le principe des folk taxonomies décrites par Brent Berlin (Tournon, 2006). De même, d'autres recherches décrivent une organisation taxonomique similaire chez les Aguaruna au Pérou (Berlin, 1976). Utilisant une méthodologie de recherche similaire, Marie Fleury conclue que le système de catégorisation de la société Aluku suit ce même principe, par contre, celle des Wayana ne peut y être raccordé (Fleury, 1999). Chez les Yanesha - comme chez les Wayãpi où une même plante possède plusieurs noms (Grenand, 2001/2002) — les plantes portent des « dénominations transitoires »: une plante définie par pare 'shemats lors d'un discours relatant un évènement morbide, peut prendre le nom de ame'taña lorsqu'elle est qualifiée de comestible. Sa qualification n'est donc pas fixe, et ne peut être modélisée de manière arborescente. Elle change au fur et à mesure du discours et des conjonctures, selon le modèle de dénomination qui, lui-même, est défini par l'ontologie de la plante. Le raisonnement diagnostique est donc vraisemblablement celui qui induit les dénominations végétales exprimant alors une entité étiologique, une pathogénie, une voie d'administration, l'origine de la plante, un remède et un mode de préparation, en somme le processus de soin à mettre en œuvre. Les modèles de dénomination des pare'shemats ne prennent donc véritablement de sens que lorsqu'ils sont regardés de manière transversale, c'est-à-dire selon la perspective du discours diagnostique.

\section{Referencias citadas}

AIKHENVALD, A. Y. \& DIXON, R. M., 2007 - Grammars in contact: a cross-linguistic typology, 375 p. ; Oxford : Oxford University Press.

BALÉE, W., 1989 - Nomenclature patterns in Ka'apor ethnbotany. Journal of ethnobiology, 9 (1) : 1-24.

BERLIN, B., 1976 - The Concept of Rank in Ethnobiological Classification: Some Evidence from Aguaruna Folk Botany. American Ethnologist, 3 : 381-99.

BERLIN, B., 1992 - Ethnobotanical classification. Principes of categorization of plants and animals in traditional societies, 335 p. ; New Jersey : Princeton University Press. 
BORIOS, S., 2005 - Att̃o yerrmuen cacac̃hno, Los yanesha y la pesca, 116 p. ; Lima, Pérou : Instituto del Bien Común.

BOURDY, G., VALADEAU, C. \& CASTILLO, J., 2008 - Yato' Ramuesh: Pare'shemats yanesha; Yato' Ramuesh: plantas medicinales yanesha, 345 p. ; Lima : PRODAPP-IRD.

CHAUMEIL, J.-P. \& CHAUMEIL, B., 1992 - L'oncle et le neveu. Journal de la Société des Américanistes, 78 (2) : 25-37.

DAIGNEAULT, A. L., 2009 - The Yanesha' (Amuesha), a Culture on the Edges of the Andes and the Amazon in Peru: An Ethnolinguistic Study of the Yanesha' Language and Speech Community, and the Traditional Role of Ponapnora, a Female Rite of Passage, 108 p. ; Montréal : Université de Montréal. Mémoire de Master, Département d'anthropologie Faculté des arts et des sciences.

DÉLÉAGE, P., 2005 - Le chamanisme sharanahua. Enquête sur l'apprentissage et l'épistémologie d'un rituel. Tome 1 Apprentissage et épistémologie, 421 p. ; Paris : Thèse d'Anthropologie Sociale, EHESS.

DESCOLA, P., 1986 - La nature domestique : Symbolisme et praxis dans l'écologie des Achuar, 450 p. ; Paris : Maison des sciences de l'homme.

DESCOLA, P., 2005 - Par-delà nature et culture, 623 p. ; Paris : Éd. Gallimard, Bibliothèque des Sciences Humaines.

DUFF-TRIPP, M., 1997 - Gramática del Idioma Yanesha' (Amuesha), 283 p. ; Lima : Instituto Linguistico de Verano.

DUFF-TRIPP, M., 1997 - Diccionario del idioma Yanesha' (Amuesha), 688 p. ; Lima : Ministerio de Educación, Instituto Lingüístico de Verano.

ERIKSON, Ph., 2004 - La face cachée de l'ancestralité. Masques et affinité chez les Matis d'Amazonie brésilienne. Journal de la société des américanistes, 90 (1) : 119-142.

ESTRELLA, E., 1981 - El mal del Arco Iris. Boletín de informaciones científicas nacionales, 16 (111) : 54.

FLEURY, M., 1999 - Dénomination et représentation des végétaux chez les Wayana et les Aluku de Guyane Française. In : Volume Régional Caraïbes (P. Grenand, éd.) : 31-44 ; Bruxelles : Programme Avenir des Peuples des Forêts Tropicales, DG VIII, Communauté Européenne.

GRENAND, F., 2001-2002 - Stratégies de nomination des plantes cultivées dans une société tupi-guarani, les Wayãpi. AMERINDIA, 26-27 : 209-247.

HIRTZEL, V., 2010 - Le Maître À Deux Têtes. Une ethnographie du rapport à soi yuracaré (Amazonie bolivienne), 742 p. ; Paris : Thèse de Doctorat, EHESS.

LENAERTS, M., 2004 - Anthropologie des Indiens Ashéninka d'Amazonie. Nos sœurs Manioc et l'étranger Jaguar, 273p. ; Paris: L'Harmattan.

LENAERTS, M., 2006 - Substances, relationships and the omnipresence of the body: an overview of Ashéninka ethnomedicine (Western Amazonia). Journal of Ethnobiology and Ethnomedicine, 2: 49. http://www.ethnobiomed.com/content/pdf/1746-42692-49.pdf

LÉVI-STRAUSS, C. \& ERIBON, D., 1988 - De près et de loin, 253 p. ; Paris : Odile Jacob.

RAMA-LECLERC, F., 2003 - Des modes de socialisation par les plantes chez les shipiboconibo d'Amazonie péruvienne, Une étude des relations entre humains et nonhumains dans la construction sociale, 555 p. ; Nanterre : Thèse de Doctorat, Université Paris $X$.

SANTOS-GRANERO, F., 2002 - Saint Christopher in the Amazon: Child Sorcery, Colonialism, and Violence among the Southern Arawak. Ethnohistory, 49 (3) : 507-543. 
SANTOS-GRANERO, F., 2006 - Sensual Vitalities: Noncorporeal Modes of Sensing and Knowing in Native Amazonia. Tipiti, 4 (I \& 2) :57-80.

SANTOS-GRANERO, F., 2011 - The virtuous Manioc and the Hormy Barbasco: Sublima and Grotesque Modes of transformation In the Origin of Yanesha Plant. Journal of ethnobiology, 31 (1) : 44-71.

SANTOS-GRANERO, F. \& BARCLAY REY DE CASTRO, F., 2004 - Guía etnográfica de la Alta Amazonía, Volumen IV, 368 p. ; Paris, Balboa : Institut français d'études andines \& Smithsonian Tropical Research Institute.

SHEPARD, G. H., 1999 - Pharmacognosy and the Senses in Two Amazonian Societies, 329 p. ; Berkeley : University of California.

SHEPARD, G. H., 2004 - A Sensory Ecology of Medicinal Plant Therapy in Two Amazonian Societies. American Anthropologist, 106 (2) : 252-266.

SMITH, R. C., 1977 - Delivrance from chaos for a song: a social and religious interpretation of the ritual performance of Amuesha music, 326 p. ; Cornell : Ph.D. Graduate School of Cornell University.

SMITH, R. C., 2004 - Where our ancestors once tread: Amuesha territoriality and sacred landscape in the Andean Amazon of Central Peru. Être Indien dans les Amériques Spoliations et résistance - Mobilisations ethniques et politiques du multiculturalisme : une perspective comparative. Colloque international de I'Université Paris III Sorbonne Nouvelle organisé par l'IHEAL-CREDAL, le CEC, I'OPEA et le CERVEPAS. Paris. 2-3 décembre

TAYLOR, A.-C., 1997 - L'oubli des morts et la mémoire des meurtres. Expériences de I'histoire chez les Jivaro. Terrain, 29 : 83-96.

TOURNON, J., 2006 - Las plantas, los rao y sus espíritus (Etnobotanica del Ucayali), 130 p. ; Lima : Gobierno Regional de Ucayali. Gerencia Regional de Desarrollo Social.

VALADEAU, C., 2010 - De l'ethnobotanique à I'articulation du soin : Approche du système nosologique chez les Yanesha de Haute Amazonie péruvienne, 382 p. ; Toulouse : Thèse de Doctorat, Université Paul Sabatier. 